\title{
LIE CONTACT STRUCTURES AND CONFORMAL STRUCTURES
}

\author{
By REIKo MiYAOKA
}

\section{§ 0. Introduction.}

In $[\mathrm{SY}]$ and $[\mathrm{M} 1]$, the notion of Lie contact structures on a $(2 n-1)$-dimensional contact manifold is established as a geometry on a manifold corresponding to the classical Lie sphere geometry [CC]. Following the connection theory by $\mathrm{N}$. Tanaka $[\mathrm{T}]$, we construct a normal Cartan connection $\omega$ (called Tanaka connection, for brevity) corresponding to the structure in [M1], which is the main tool to solve the equivalence problem (see [SY]).

A typical and important example of the structure exists on the unit tangent bundle $T_{1} M$ of an $n$-dimensional Riemannian manifold $M$. In this paper, we calculate the curvature $K$ of Tanaka connection of this structure on $T_{1} M$. We call $K$ the Lie curvature of $T_{1} M$. In particular, when $K \equiv 0, T_{1} M$ is called Lie flat, and is locally Lie equivalent to the model space $=T_{1} S^{n}$, the unit tangent bundle of the standard $n$-sphere [SY]. This is apparently the case when $M$ is conformally flat $(\S 1)$. The inverse problem is presented by Sato [S]: Is $M$ conformally flat when $T_{1} M$ is Lie flat?

The purpose of this paper is to answer this problem affirmatively. The description of Tanaka connection and its curvature for this structure is given in Theorem in $\S 5$, where the Lie curvature is expressed in terms of all coefficients of Weyl's conformal curvature. As a result, we know that the structure depends only on the conformal structure of $M$, and moreover we obtain

Corollary 1. Let $M$ be a Riemannian manifold of $\operatorname{dim} \geqq 3$. Then $M$ is conformally flat if and only if $T_{1} M$ is Lie flat.

CoRollary 2. Let $M$ and $M^{\prime}$ be two Riemannian manifolds of $\operatorname{dim} \geqq 3$. Let $\tilde{f}: T_{1} M \rightarrow T_{1} M^{\prime}$ be a bundle map which preserves the Lie curvature. Then the induced map $f: M \rightarrow M^{\prime}$ preserves the conformal curvature.

A resume of [M1] and the present paper is given in [M2].

The author would like to express her hearty thanks to Professors H. Sato and K. Yamaguchi for their valuable suggestions.

Received June 14, 1989; Revised April 26, 1990. 


\section{§. Preliminaries.}

In this paper, we follow the argument in [M1] and use the notations in it.

Let $\boldsymbol{R}_{2}^{n+3}=\left\{x=\left(x^{0}, \cdots, x^{n+2}\right), x^{2} \in \boldsymbol{R}\right\}$ be an $(n+3)$-dimensional real vector space endowed with a scalar product $\langle$,$\rangle with signature (+, \cdots,+,-,-)$ and let $\boldsymbol{R}_{1}^{n+2}=\left\{x \in \boldsymbol{R}_{2}^{n+3}, x^{n+2}=0\right\}$. Denote by $P^{n+2}$ and $P^{n+1}$ the associated projective spaces. Furthermore, let $\boldsymbol{R}^{n+1}=\left\{x \in \boldsymbol{R}_{1}^{n+2}, x^{n+1}=0\right\}$ be the $(n+1)$-dimentional space-like subspace of $\boldsymbol{R}_{1}^{n+2}$. By $\langle$,$\rangle , we denote the induced scalar product on$ $\boldsymbol{R}_{1}^{n+2}$ or on $\boldsymbol{R}^{n+1}$. Now, the unit sphere $S^{n}=\left\{x \in \boldsymbol{R}^{n+1} \mid\langle x, x\rangle=1\right\}$ is naturally embedded in $P^{n+1}$ as a Möbius space $Q^{n}$,

$$
S^{n} \cong Q^{n}=\left\{[y] \in P^{n+1} \mid\langle y, y\rangle=0\right\},
$$

by $x \rightarrow(x, 1) \in \boldsymbol{R}_{1}^{n+2}$. On the other hand, let $\Sigma$ be the set of all oriented hyperspheres in $S^{n} ; \Sigma=\left\{(m, \theta) \in S^{n} \times[0, \pi) \mid\right.$ an oriented hypersphere with center $m$ and radius $\theta\}$. Then $\Sigma$ is naturally embedded in $P^{n+2}$ as a quadratic $Q^{n+1}$,

$$
\Sigma \cong Q^{n+1}=\left\{[k] \in P^{n+2} \mid\langle k, k\rangle=0\right\},
$$

by $(m, \theta) \rightarrow(m, \cos \theta, \sin \theta) \in \boldsymbol{R}_{2}^{n+3}$.

The Möbius group $L$ is, by definition, a group consists of projective transformations of $P^{n+1}$ preserving $Q^{n}$, and we have $L=P O(n+1,1)$. The Lie transformation group $G$ is, by definition, a group consists of projective transformations of $P^{n+2}$ preserving $Q^{n+1}$, and we get $G=P O(n+1,2)$. Clearly we have $L \subset G$. Now, let $\Lambda^{2 n-1}=\left\{\right.$ lines in $Q^{n+1}$ generated by $\left(\left[k_{1}\right],\left[k_{2}\right]\right) \in Q^{n+1} \times Q^{n+1}$, $\left.\left\langle k_{1}, k_{2}\right\rangle=0\right\}$. Then we have

$$
T_{1} S^{n}=\left\{(u, v) \in S^{n} \times S^{n} \mid\langle u, v\rangle=0\right\} \cong \Lambda^{2 n-1}
$$

under a mapping $(u, v) \rightarrow\left(\left[k_{1}\right],\left[k_{2}\right]\right)$, where $k_{1}=(u, 1,0), k_{2}=(v, 0,1)$. Since $G$ preserves $\langle$,$\rangle , it induces an action on \Lambda^{2 n-1}$. This action resticted to $L$ is translated as follows: A Möbius transformation $\sigma: S^{n} \rightarrow S^{n}$ is lifted to Lie transformations $\sigma_{ \pm}: T_{1} S^{n} \rightarrow T_{1} S^{n}$, by

$$
\sigma_{ \pm}(X)= \pm \sigma_{*} X /\left\|\sigma_{*} X\right\|
$$

We denote the subgroup $\sigma_{+}(L)$ of $G$ by $G_{M}$. It is easy to see that $G_{M}$, and so $G$ acts on $\Lambda^{2 n-1}$ transitively. Let $G_{M}^{\prime}$ and $G^{\prime}$ be isotropy subgroups:

FACT 1.1. $\Lambda^{2 n-1}=G / G^{\prime}=G_{M} / G_{M}^{\prime}$.

As is shown in [M1], the Lie algebra $\mathfrak{g}$ of $G$ is given by

$$
\begin{aligned}
& \mathrm{g}=\sum_{p=-2}^{2} g_{p}, \quad\left[g_{\imath}, g_{\jmath}\right]=g_{\imath+\jmath}, \\
& g_{-2}={ }^{t} g_{2}=\left\{\left(\begin{array}{lll}
0 & 0 & c_{p} \\
0 & 0 & 0 \\
0 & 0 & 0
\end{array}\right), c_{p}=\left(\begin{array}{rr}
0 & p \\
-p & 0
\end{array}\right)\right\},
\end{aligned}
$$




$$
\begin{aligned}
& \mathfrak{g}_{-1}={ }^{t} \mathfrak{g}_{1}=\left\{\left(\begin{array}{ccc}
0 & b & 0 \\
0 & 0 & { }^{t} b \\
0 & 0 & 0
\end{array}\right),{ }^{t} b \in \boldsymbol{R}^{n-1} \times \boldsymbol{R}^{n-1}\right\}, \\
& \mathfrak{g}_{0}=\left\{\left(\begin{array}{ccc}
a & 0 & 0 \\
0 & e & 0 \\
0 & 0 & -{ }^{t} a
\end{array}\right), a \in \mathfrak{g l}(2, \boldsymbol{R}), e \in \mathfrak{D}(n-1)\right\} .
\end{aligned}
$$

Here, note that a base of $\boldsymbol{R}_{2}^{n+3}$ is chosen so that

$$
\langle u, v\rangle=-2 u^{0} v^{n+1}-2 u^{1} v^{n+2}+\sum_{\imath=2}^{n} u^{2} v^{\imath},
$$

for $u=\left(u^{0}, u^{1}, \cdots, u^{n+2}\right)$ and $v=\left(v^{0}, v^{1}, \cdots, v^{n+2}\right) \in \boldsymbol{R}_{2}^{n+3}$. Thus we have $\boldsymbol{R}_{1}^{n+2}=$ $\left\{u \in \boldsymbol{R}_{2}^{n+3}, u^{n+2}=-(1 / 2) u^{1}\right\}$, and $\boldsymbol{R}^{n+1}=\left\{u \in \boldsymbol{R}_{1}^{n+2}, u^{n+1}=-(1 / 2) u^{0}\right\}$. We may assume that $G_{M}=\left\{h \in G \mid h\right.$ preserves $\left.\boldsymbol{R}_{1}^{n+2}\right\}$. Then the Lie algebra $g_{M}$ of $G_{M}$ is given by

$$
\mathfrak{g}_{M}=\left\{\left(\begin{array}{ccc}
a & b & c_{p} \\
d & e & { }^{t} b \\
{ }^{t} c_{q} & { }^{t} d & -{ }^{t} a
\end{array}\right) \in \mathfrak{g}, a=\left(\begin{array}{cc}
* & -\frac{1}{2} p \\
-2 q & 0
\end{array}\right), d=\left(d_{1}, d_{2}\right),{ }^{t} b=\left({ }^{t} b_{1},-2^{t} d_{2}\right)\right\} .
$$

Now, we have $\mathfrak{g}^{\prime}=\mathfrak{g}_{0}+\mathfrak{g}_{1}+\mathfrak{g}_{2}, \mathfrak{g}_{M}^{\prime}=\mathfrak{g}^{\prime} \cap \mathfrak{g}_{M}$, where $\mathfrak{g}^{\prime}$ and $\mathfrak{g}_{M}^{\prime}$ are Lie algebras of $G^{\prime}$ and $G_{M}^{\prime}$, respectively. Note that $\mathfrak{D}(n-1) \subset \mathfrak{c}(n-1) \subset \mathfrak{g}_{0} \cap \mathfrak{g}_{M} \subset \mathfrak{g}_{M}^{\prime} \subset \mathfrak{g}^{\prime}$. From these facts, we get

FACT 1.2. [see Lemma 1.2, M1].

$$
\begin{aligned}
& G^{\prime}=\left\{h=\left(\begin{array}{ccc}
A & 0 & 0 \\
g d & g & 0 \\
{ }^{t} A^{-1}\left\{\frac{1}{2} t d d+f\right\} & { }^{t} A^{-1 t} d & { }^{t} A^{-1}
\end{array}\right), \begin{array}{l}
g \in O(n-1) \quad A \in G L(2, \boldsymbol{R}) \\
d \in \boldsymbol{R}^{n-1} \times \boldsymbol{R}^{n-1}, f=\left(\begin{array}{cr}
0-q \\
q & 0
\end{array}\right)
\end{array}\right\}, \\
& G_{M}^{\prime}=\left\{h \in G^{\prime} \mid A=\left(\begin{array}{ll}
\alpha & 0 \\
\gamma & 1
\end{array}\right), g d=(*, 0), \alpha \neq 0\right\},
\end{aligned}
$$

and $O(n-1) \subset C O(n-1) \subset G_{M}^{\prime} \subset G^{\prime}$.

Put, $\mathfrak{m}=T_{0}\left(G / G^{\prime}\right), \quad \tilde{G}=\rho\left(G^{\prime}\right)$ and $\tilde{G}_{M}=\rho\left(G_{M}^{\prime}\right)$, where $\rho: G^{\prime}, G_{M}^{\prime} \rightarrow G L(\mathfrak{m})=$ $G L(2 n-1)$ is the linear isotropy representation. Since $\operatorname{Ker} \rho=\exp \mathrm{g}_{2}$, denoting $\rho(O(n-1))=O(n-1)$ and $\rho(C O(n-1))=C O(n-1)$, we get

FACT 1.3. [see Proposition 1.3, M1]. 


$$
\tilde{G}=\left\{\left(\begin{array}{ccc}
\operatorname{det} A & 0 & 0 \\
* & g \otimes A \\
* &
\end{array}\right)\right\}, \quad \tilde{G}_{M}=\left\{\left(\begin{array}{ccc}
\alpha & 0 & 0 \\
0 & \alpha g & 0 \\
* & \gamma g & g
\end{array}\right)\right\},
$$

where $A, g, \alpha, \gamma$ are given in Fact 1.2 , and

$$
O(n-1) \subset C O(n-1) \subset \tilde{G}_{M} \subset \tilde{G} .
$$

Now, let $N$ be a $(2 n-1)$-dimensional contact manifold. It is well-known that the linear frame bundle $L(N)$ has a reduction $L^{\#}(N)$ with structure group $G_{0}(\mathfrak{m})^{\#}=\left\{\left(\begin{array}{lc}a & 0 \\ \zeta & C S p(n-1, R)\end{array}\right), a \neq 0\right\} . \quad$ Noting that $\tilde{G} \subset G_{0}(\mathfrak{m})^{\#},[\mathrm{M} 1]$, we define:

Definition. A $\tilde{G}$-reduction of $L^{\#}(N)$ is called a Lie contact structure on $N$.

Now, recall the way of construction of Lie contact structure on the unit tangent bundle $T_{1} M$ of an $n$-dimensional riemannian manifold $(M, g)$. Let $Q_{g}$ be the principal fibre bundle over $M$ with structure group $O(n)$. According to [KN, p. 57], $P_{g}=\left(Q_{g} / O(n-1), O(n-1)\right)$ is a principal fibre bundle over $T_{1} M$ with structure group $O(n-1)$. It is shown in [M1] that the extended bundle

$$
\tilde{P}_{g}=Q_{g} \times_{o(n-1)} \tilde{G},
$$

gives a Lie contact structure on $T_{1} M$.

It is obvious that $T_{1} M$ is Lie flat if $M$ is conformally flat, since a conformal transformation is lifted to a Lie transformation by $(*)$. But it is a non-trivial matter to see whether $M$ is conformally flat when $T_{1} M$ is Lie flat, since the structure group is enlarged. The purpose of this paper is to solve this question.

For later use, recall the geometry of the unit tangent bundle $T_{1} M$ of an $n$-dimensional riemannian manifold $M$. Let $z_{1} \in T_{1} M$ and let $\left(z_{1}, \cdots, z_{n}\right)$ be an orthonormal frame of $M$ at $p=\pi_{1}\left(z_{1}\right) \in M$, where $\pi_{1}: T_{1} M \rightarrow M$ is the projection. By using the horizontal lift $z_{\imath}^{h}$ and the vertical lift $z_{\imath}^{v}$ of $z_{i} \in T_{p} M$ to $T_{z_{1}} T M$, we make a frame $u(z)=\left(u_{1}, \cdots, u_{2 n-1}\right)$ of $T_{1} M$ at $z_{1}$, where $u_{i}=z_{\imath}^{h}, 1 \leqq i \leqq n$, and $u_{n+\imath-1}=z_{\imath}^{v}, 2 \leqq i \leqq n$. Note that $z_{1}^{v}$ is a normal vector of $T_{1} M$ in $T M$. It is wellknown that $u(z)$ is an orthonormal frame of $T_{z_{1}} T_{1} M$ with respect to the metric on $T_{1} M$ induced from the Sasaki metric on $T M$. Now, let $h \in O(n-1)$ and put $\tilde{h}=\left(\begin{array}{ll}1 & 0 \\ 0 & h\end{array}\right) \in O(n)$. We make $h$ act on $u(z)$ by

$$
u(z) h=u(z \tilde{h}) .
$$

Then we obtain an $O(n-1)$-bundle $\pi: P_{g} \rightarrow T_{1} M$, where $P_{g}=\left\{u(z) \mid z=\left(z_{2}\right)\right.$ is an orthonormal frame of $M$ at $\left.\pi_{1} \circ \pi(z)=\pi_{1}\left(z_{1}\right)\right\}$. We have shown in the end of the proof of [M1, Proposition 2.3] that $u(z)$ is a frame adapted to the Lie contact structure. 


\section{$\S 2$. Construction of a normal Cartan connection $(Q, \chi)$.}

In this section, following the argument in [M1], we construct a normal Cartan connection of type $H / H_{0}$ on an $H_{0}$-reduction $(Q, \zeta)$ of the Lie contact structure $\widetilde{P}$ over $T_{1} M$, when $M$ is a Rimannian manifold. Let $\pi: \tilde{P}_{\rightarrow} T_{1} M$ be the projection. Here, we start with the $O(n-1)$-reduction $P_{g}$ of $\tilde{P}$.

Let $\mathfrak{f}=\mathfrak{m}+\mathfrak{p}(n-1)$, where $\mathfrak{p}(n-1)$ is the Lie algebra of $O(n-1)$, and let $K$ be a Lie subgroup of $G$ of which Lie algebra is $¥$. As is mentioned in $\S 1$, an element $u(z) \in P_{g}$ is an orthonormal base of $T_{z_{1}} T_{1} M$, at $z_{1}=\pi(u(z))$ with respect to the metric $s_{g}$ induced from the Sasaki metric $s_{g}$ on $T M$. Therefore, as a basic form on $P_{g}$, we should take

$$
\begin{array}{ll}
\zeta^{i}(X)=s_{g}\left(\pi_{*} X, u_{\imath}\right), & 1 \leqq i \leqq n, \\
\zeta^{\bar{i}}(X)=s_{g}\left(\pi_{*} X, u_{\bar{\imath}}\right), & 2 \leqq i \leqq n,
\end{array}
$$

where $X \in T_{u(z)} P_{g}$ and we put $u_{i}=u_{n+\imath-1}, 2 \leqq i \leqq n$. We will express them in a local coordinate of $P_{g}$. Around $u_{0}=u\left(z_{0}\right) \in P_{g}$, where $z_{0}=\left(z_{1}, \cdots, z_{n}\right), \pi\left(u_{0}\right)=z_{1}$, and $\pi_{1}\left(z_{1}\right)=p \in M$, we choose a local coordinate $\left(x^{2}, z_{j}^{i}\right), 1 \leqq i, j \leqq n$, as follows: let $\left(x^{1}, \cdots, x^{n}\right)$ be the geodesic normal coordinate of $M$ around $p$ such that $z_{i}(p)=\partial / \partial x^{2}$, and let $\left(z_{\jmath}^{i}\right) \equiv G L(n, \boldsymbol{R})$ be such that

$$
g_{i j} z_{k}^{2} z_{m}^{j}=\delta_{k m},
$$

where $g_{\imath \jmath}$ is the component of the Riemannian metric $g$ on $M$ with respect to $\left(x^{1}, \cdots, x^{n}\right)$. Here and hereafter, we use Einstein convension for $1 \leqq i, j, k, m$, $r, s, t, u, v \leqq n$, unless otherwise stated. Note that we have

$$
\left\{\begin{array}{l}
z_{j}^{2}(p)=\delta_{j}^{\imath}, \\
g_{\imath j}(p)=\delta_{i \jmath}, \\
\frac{\partial}{\partial x^{k}} g_{\imath j}(p)=0, \\
\left\{\begin{array}{c}
i \\
j k
\end{array}\right\}(p)=0,
\end{array}\right.
$$

where $\left\{\begin{array}{c}i \\ j k\end{array}\right\}$ is the Christoffel's symbol. Let $\left(x^{i}, v^{i}\right)$ be a local coordinate of $T M$ expressing $v^{2}\left(\partial / \partial x^{i}\right) \in T_{(x \imath)} M$, and $\left(x^{2}, v^{2}, \xi^{2}, \eta^{i}\right)$ be a coordinate of $T T M$ expressing $\xi^{\imath}\left(\partial / \partial x^{i}\right)+\eta^{\imath}\left(\partial / \partial v^{i}\right) \in T_{(x i, v \imath)} T M$. The coeffecients of the Sasaki metric ${ }_{g} s$ on $T M$ are then given by [SS]

$$
\begin{aligned}
& G_{\imath \jmath}=g_{\imath j}+g_{r u}\left\{\begin{array}{c}
r \\
s i
\end{array}\right\}\left\{\begin{array}{c}
u \\
t j
\end{array}\right\} v^{s} v^{t}, \\
& G_{\imath n+\jmath}=g_{r j}\left\{\begin{array}{c}
r \\
u i
\end{array}\right\} v^{u},
\end{aligned}
$$




$$
G_{n+\imath n+j}=g_{\imath \jmath} .
$$

Since we can express $u \in P_{g}$ in a neighbourhood of $u_{0}$ by

$$
\begin{cases}u_{i}=\left(z_{\imath}^{3},-\left\{\begin{array}{c}
j \\
s t
\end{array}\right\} z_{1}^{s} z_{\imath}^{t}\right), & 1 \leqq i \leqq n \\
u_{i}=\left(0, z_{\imath}^{j}\right), & 2 \leqq i \leqq n\end{cases}
$$

we can take $\left(x^{2}, z_{j}^{i}\right), 1 \leqq i, j \leqq n$, satisfying (2.1) as a local coordinate of $P_{g}$. Moreover, in this local coordinate, we can show easily that the basic forms are expressed by

$$
\begin{aligned}
& \zeta^{i}=g_{j k} z_{i}^{k} d x^{j}, \quad 1 \leqq i \leqq n, \\
& \zeta^{\bar{i}}=g_{j k} z_{i}^{k}\left(d z_{1}^{j}+\left\{\begin{array}{c}
j \\
s t
\end{array}\right\} z_{1}^{s} d x^{t}\right), \quad 2 \leqq i \leqq n .
\end{aligned}
$$

Now, define

$$
\chi_{r}^{\prime i}=g_{j k} z_{i}^{k}\left(d z_{r}^{j}+\left\{\begin{array}{c}
j \\
s t
\end{array}\right\} z_{r}^{s} d x^{t}\right), \quad 2 \leqq i, r \leqq n,
$$

and put $\chi^{\prime \imath}=\zeta^{i}, 1 \leqq i \leqq n, \chi^{\prime i}=\zeta^{\bar{i}}, 2 \leqq i \leqq n$.

LEMMA 2.1. $\chi^{\prime}$ is a Cartan connection of type $K / O(n-1)$ on $P_{g}$.

Proof. Obviously, $\chi^{\prime}$ is an $\mathfrak{f}^{\prime}$-valued 1 -form on $P_{g}$. Then $\chi^{\prime}$ is a Cartan connection of type $K / O(n-1)$ iff

C1) For $X \in T P_{g}, \chi^{\prime}(X)=0$ implies $X=0$.

C2) $\chi^{\prime}\left(A^{*}\right)=A, A \in \mathfrak{p}(n-1)$ and $A^{*}$ is the fundamental vector field.

C3) $R_{a}^{*} \chi^{\prime}=A d\left(a^{-1}\right) \chi^{\prime}, a \in O(n-1)$.

For $X=\left(d x^{2}, d z_{\jmath}^{i}\right) \in T_{u_{0}} P_{g}$, we have $\chi^{\prime 2}(X)=d x^{\imath}, \chi^{\prime i}(X)=d z_{1}^{2}, \chi_{r}^{\prime 2}(X)=d z_{r}^{2}$, and so $\mathrm{C} 1)$ is obvious. For $A=\left(A_{r}^{i}\right) \in \mathfrak{p}(n-1)$, put $a_{t}=\exp t A=\left(a_{j}^{\imath}(t)\right) \in O(n-1)$. We use the Einstein convension over $2 \leqq i, j \leqq n$ as far as $a_{j}^{2}(t)$ is concerned. Since $u_{0} a_{t}=u\left(z_{0} \tilde{a}_{t}\right)$ by (1.2), the local coordinate expression of $A_{u_{0}}^{*}$ is given by $d x^{2}=0$, $d z_{1}^{2}=0$, and $d z_{r}^{2}=\left(d a_{r}^{i}\right)(0)=A_{r}^{2}$, and we get $\chi^{\prime}\left(A_{u_{0}}^{*}\right)=A$. Now, for $a=\left(a_{j}^{i}\right) \in O(n-1)$ and $X=\left(d x^{2}, d z_{j}^{i}\right) \in T_{u_{0}} P_{g}$, from $R_{a}^{*} \chi^{\prime}(X)=\chi^{\prime}\left(R_{a *} X\right)$ and $R_{a *} X=\left(d x^{2}, d z_{1}^{2}, d\left(z_{k}^{j} a_{r}^{k}\right)\right)$, $1 \leqq i \leqq n, 2 \leqq j, r \leqq n$ at $u_{0} a \in P_{g}$, it follows

$$
\begin{aligned}
& \zeta^{1}\left(R_{a *} X\right)=d x^{1}, \\
& \zeta^{i}\left(R_{a *} X\right)=\sum_{k=2}^{n} a_{i}^{k} d x^{k}, \quad 2 \leqq i \leqq n, \\
& \zeta^{\bar{i}}\left(R_{a *} X\right)=\sum_{k} a_{i}^{k} d z_{1}^{k}, \quad 2 \leqq i \leqq n,
\end{aligned}
$$




$$
\chi_{r}^{\prime i}\left(R_{a *} X\right)=\sum_{j, k} a_{i}^{\jmath}\left(d z_{k}^{j}\right) a_{r}^{k}, \quad 2 \leqq i, r \leqq n .
$$

On the other hand $A d\left(a^{-1}\right) \chi^{\prime}(X)=a^{-1} \chi^{\prime}(X) a={ }^{t} a \chi^{\prime}(X) a$ is given by

$$
\left(\begin{array}{ccc}
I_{2} & 0 & 0 \\
0 & { }^{t} a & 0 \\
0 & 0 & I_{2}
\end{array}\right)\left(\begin{array}{ccc}
0 & \hat{\zeta} & \hat{\zeta}^{1} \\
0 & \hat{\chi}^{\prime} & { }^{t} \hat{\zeta} \\
0 & 0 & 0
\end{array}\right)\left(\begin{array}{ccc}
I_{2} & 0 & 0 \\
0 & a & 0 \\
0 & 0 & I_{2}
\end{array}\right)=\left(\begin{array}{ccc}
0 & \hat{\zeta} a & \hat{\zeta}^{1} \\
0 & { }^{t} a \hat{\chi}^{\prime} a & { }^{t} a^{t} \hat{\zeta} \\
0 & 0 & 0
\end{array}\right),
$$

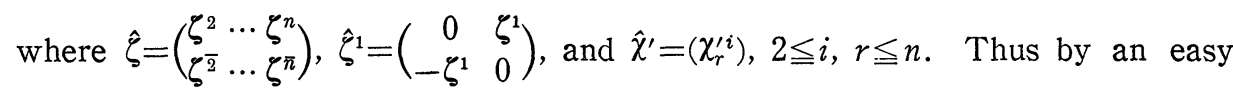
calculation, we get $\mathrm{C} 3$ ).

q.e.d.

Now, enlarging the structure group to $H_{0}=\left\{a \in G_{0} \mid \operatorname{det} a= \pm 1\right\}$, we get a principal fibre bundle $Q=P_{g} \times_{o(n-1)} H_{0}$ over $T_{1} M$. A local coordinate of $Q$ is given by $\left(x^{\imath}, z_{\jmath}^{\imath}, h_{b}^{a}\right)$, where $\left(h_{b}^{a}\right) \in \pm S L(2, \boldsymbol{R})$, since $H_{0} / O(n-1) \cong \pm S L(2, \boldsymbol{R})$. Denote by $\tilde{\chi}^{\prime}$ the Cartan connection on $Q$ naturally extended from $\chi^{\prime}$ on $P_{g}$, that

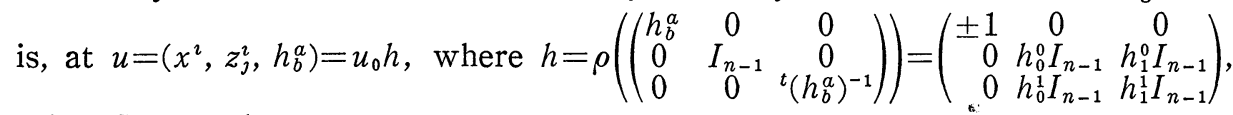
using C3), we have

$$
\begin{aligned}
& \tilde{\zeta}^{1}=\zeta^{1}, \\
& \tilde{\zeta}^{i}=h_{1}^{1} \zeta^{i}-h_{1}^{0} \zeta^{\bar{i}}, \\
& \tilde{\zeta}^{\bar{i}}=-h_{0}^{1} \zeta^{i}+h_{0}^{0} \zeta^{\bar{i}}, \\
& \left(\tilde{\chi}_{r}^{\prime i}\right)=\left(\chi_{r}^{\prime}\right), \\
& \left(\tilde{\chi}_{0}^{\prime a}\right)=\left(\begin{array}{cc}
(s v+t u) \chi_{0}^{\prime 0}+u v \chi_{1}^{\prime 0}-s t \chi_{0}^{\prime 1} & 2 t v \chi_{0}^{\prime 0}+v^{2} \chi_{1}^{\prime 0}-t^{2}{\chi_{0}^{\prime 1}}_{0}^{\prime} \\
-2 s u \chi_{0}^{\prime 0}-u^{2} \chi_{1}^{\prime 0}+s^{2} \chi_{0}^{\prime 1} & -(t u+s v) \chi_{0}^{\prime 0}-u v \chi_{1}^{\prime 0}+s t{\chi_{0}^{\prime}}_{0}^{\prime}
\end{array}\right),
\end{aligned}
$$

for $(a, b)=(0,0),(0,1)$ and $(1,0)$, where

$$
\chi_{b}^{\prime a}=d h_{b}^{a},
$$

putting $\left(\begin{array}{ll}s & t \\ u & v\end{array}\right)=\left(h_{b}^{a}\right)$, and $\zeta^{\gamma}$ and $\chi^{\prime}$ are evaluated at $u_{0}$. In the following, we use the notation $\chi^{\prime}$ instead of $\tilde{\chi}^{\prime}$ for simplicity. Let $\Psi^{\prime}$ be the curvature form of $\chi^{\prime}$ and put $\Psi^{\prime}=(1 / 2) T^{\prime} \zeta \wedge \zeta$. With respect to the base of $g$ given in [M1, §3], the $\mathrm{g}_{p}$-component $T_{p}^{\prime}$ is given by $T_{-2}^{\prime}=T_{\beta \gamma}^{\prime 1} e_{1}, T_{-1}^{\prime}=T_{\beta \gamma}^{\prime i} e_{i}+T_{\beta \gamma}^{\prime i} e_{i}, 2 \leqq i \leqq n$, and $T_{0}^{\prime}=T_{r \beta \gamma}^{\prime i} e_{r}^{2}+T_{0 \beta \gamma}^{\prime 0} e_{0}^{0}+T_{1 \beta \gamma}^{\prime 0} e_{1}^{0}+T^{\prime 1}{ }_{0 \beta \gamma} e_{0}^{1}, 2 \leqq i, r \leqq n$, and $\beta, \gamma \in\{1, \cdots, n, \overline{2}, \cdots, \bar{n}\}$.

Proposition 2.2. The curvature $T^{\prime}$ of $\chi^{\prime}$ at $u_{0} \in P_{g}$ is given by

$$
\begin{aligned}
& T_{-2}^{\prime}=0, \\
& T_{1 j}^{\prime i_{j}}=\delta_{\jmath}^{i}, \quad T_{\beta \gamma}^{\prime i}=0 \text { otherwise, }
\end{aligned}
$$




$$
\begin{aligned}
& T_{1 j}^{\prime \bar{i}}=R_{11 \jmath}^{\imath}, \quad T_{j k}^{\prime \bar{i}}=R_{1 j k}^{\imath}, \quad T_{j \gamma}^{\prime \bar{i}}=0, \\
& T^{\prime i}{ }_{j s t}^{i}=R_{j s t}^{i}, \quad T^{\prime i}{ }_{j \bar{k} \bar{m}}=\delta_{k}^{i} \delta_{{ }_{j}}-\delta_{m}^{i} \delta_{j k}, \quad T_{j \beta \gamma}^{\prime i}=0 \text { otherwise, } \\
& T^{\prime 0}{ }_{0 \beta \gamma}=T^{\prime 0}{ }_{1 \beta \gamma}=T^{\prime 1}{ }_{0 \beta \gamma}=0 \text {, }
\end{aligned}
$$

where $i, j, k \in\{2, \cdots, n\}, s, t \in\{1, \cdots, n\}, \beta, \gamma \in\{1, \cdots, n, \overline{2}, \cdots, \bar{n}\}$, and $R_{j k m}^{2}$ denotes the coefficients of the Riemannian curvature of the base manifold $M$ with respect to $\left(x^{i}\right)$ at $p=\pi_{1} \circ \pi\left(u_{0}\right)$.

To prove this, we prepare:

LEMMA 2.3. We have the following formulas:

$$
\begin{aligned}
& d x^{i}=z_{j}^{i} \zeta^{j}, \quad \text { and at } u_{0}, \quad d x^{2}=\zeta^{i}, \quad 1 \leqq i \leqq n, \\
& d z_{1}^{2}=z_{j}^{i} \zeta^{j}-\left\{\begin{array}{c}
i \\
s t
\end{array}\right\} z_{1}^{s} d x^{t}, \text { and at } u_{0}, \quad d z_{1}^{2}=\zeta^{\bar{i}}, \quad 2 \leqq i \leqq n, \\
& d z_{r}^{i}=z_{j}^{i} \chi_{r}^{\prime}-\left\{\begin{array}{c}
i \\
s t
\end{array}\right\} z_{1}^{s} z_{r}^{t}, \quad \text { and at } u_{0}, \quad d z_{r}^{2}=\chi_{r}^{\prime i}, \quad 2 \leqq i, r \leqq n, \\
& 0=d z_{j}^{i}+d z_{i}^{j} \quad \text { at } u_{0}, \quad 1 \leqq i \leqq n, \\
& 0=\partial_{k} z_{j}^{i} \quad \text { for } 1 \leqq j<i \leqq n \text { and at } u_{0} \text { for } 1 \leqq i, j, k \leqq n, \\
& \partial_{\bar{r}} z_{1}^{2}=\delta_{r}^{i}, \quad 2 \leqq i, r \leqq n, \\
& \partial_{\bar{r}}\left(z_{\jmath}^{i}\right)=\delta_{r}^{i} \delta_{j 1}-\delta_{r j} \delta_{1}^{i} \text { at } u_{0} \text { for } 1 \leqq i, j \leqq n, \quad 2 \leqq r \leqq n,
\end{aligned}
$$

where we use $\partial_{i}=\partial / \partial x^{2}$ and $\partial_{\bar{r}}=\partial / \partial z_{1}^{r}$.

Proof. Since $g_{j k} z_{i}^{k} z_{m}^{j}=\delta_{i m},\left(y_{j}^{i}\right)$ given by $y_{j}^{\imath}=g_{j k} z_{\imath}^{k}$ is the inverse matrix of $\left(z_{j}^{i}\right)$. The first three are direct consequence of this fact and (2.2). From $0=$ $d\left(g_{i j} z_{k}^{2} z_{m}^{j}\right)=\delta_{i j}\left\{\left(d z_{k}^{i}\right) \delta_{m}^{j}+\delta_{k}^{2} d z_{m}^{j}\right\}$ at $u_{0}$ follows (2.8). Since we may consider $z_{j}^{\imath}$, $1 \leqq j<i$, as free variables, we have $\partial_{k}\left(z_{\jmath}^{i}\right) \equiv 0$, for $1 \leqq k \leqq n, 1 \leqq j<i \leqq n$. Especially at $u_{0}$, by virtue of (2.8), we get (2.9). In the same way, since $\partial_{\bar{r}}=\partial / \partial z_{1}^{r}, 2 \leqq r$ $\leqq n$, we get (2.10) for $2 \leqq i, r \leqq n$, and $\partial_{\bar{r}} z_{j}^{2} \equiv 0$ for $2 \leqq i<j \leqq n$. The last formula follows from (2.8).

q.e.d.

Proof of Proposition 2.2. Since $\chi_{b}^{\prime a}=d h_{b}^{a},(a, b)=(0,0),(0,1),(1,0)$, and since $\Psi^{\prime}=(1 / 2) T^{\prime} \zeta \wedge \zeta$, we may ignore the terms $\chi_{b}^{\prime a}$ in the structure equation (see (3.1) in $\S 3$ ), when we calculate the curvature. It is ovbious that $T_{b \beta \gamma}^{\prime a}=0$. Now, we obtain

$$
\begin{aligned}
\Psi^{\prime 1} & =d \zeta^{1}+\sum_{i=2}^{n} \zeta^{i} \wedge \zeta^{i} \\
& =d\left(g_{j k} z_{1}^{k} d x^{j}\right)+\sum_{i=2}^{n} d x^{i} \wedge d z_{1}^{i}
\end{aligned}
$$




$$
\begin{aligned}
& =\sum_{j=1}^{n} d z_{1}^{\jmath} \wedge d x^{\jmath}+\sum_{i=2}^{n} d x^{\imath} \wedge d z_{1}^{2} \\
& =0
\end{aligned}
$$

i.e. $T_{-2}^{\prime}=0$. Next, we get

$$
\begin{aligned}
\Psi^{\prime \prime} & =d \zeta^{i}+\sum_{r=2}^{n} \chi_{r}^{\prime i} \wedge \zeta^{r} \\
& =d\left(g_{j k} z_{i}^{k} d x^{j}\right)+\sum_{r=2}^{n} g_{j k} z_{i}^{k}\left(d z_{r}^{j}+\left\{\begin{array}{c}
j \\
s t
\end{array}\right\} z_{1}^{s} d x^{t}\right) \wedge \zeta^{r} \\
& =\sum_{j=1}^{n} d z_{\imath}^{j} \wedge d x^{\jmath}+\sum_{r=2}^{n} d z_{r}^{i} \wedge d x^{r} \\
& =d z_{\imath}^{1} \wedge d x^{1} \\
& =-\zeta^{i} \wedge \zeta^{1}, \quad i \geqq 2,
\end{aligned}
$$

i.e. $T_{1 \bar{i}}^{\prime}=1$ and $T_{\beta \gamma}^{\prime i}=0$ otherwise. From

$$
\begin{aligned}
\Psi^{\prime \bar{i}} & =d \zeta^{\bar{i}}+\sum_{r=2}^{n} \chi_{r}^{\prime i} \wedge \zeta^{\bar{r}} \\
& =d\left(g_{j k} z_{i}^{k}\left(d z_{1}^{j}+\left\{\begin{array}{c}
j \\
s t
\end{array}\right\} z_{1}^{s} d x^{t}\right)\right)+\sum_{r=2}^{n} d z_{r}^{i} \wedge d z_{1}^{r} \\
& =\sum_{j=1}^{n} d z_{2}^{j} \wedge d z_{1}^{j}+d\left\{\begin{array}{c}
i \\
1 t
\end{array}\right\} \wedge d x^{t}+\sum_{r=2}^{n} d z_{r}^{i} \wedge d z_{1}^{r} \\
& =\left(\partial_{s}\left\{\begin{array}{c}
i \\
1 t
\end{array}\right\}\right) d x^{s} \wedge d x^{t}, \quad i \geqq 2,
\end{aligned}
$$

follow $T_{s t}^{\prime \bar{i}}=R_{1 s t}^{2}$ and $T_{j i}^{\prime \bar{i}}=0$. Now, we have

$$
\begin{aligned}
\Psi_{r}^{\prime}{ }_{r} & =d \chi_{r}^{\prime i}+\sum_{k=2}^{n} \chi_{k}^{\prime i} \wedge \chi_{r}^{\prime k} \\
& =d\left(g_{j k} z_{i}^{k}\left(d z_{r}^{j}+\left\{\begin{array}{c}
j \\
s t
\end{array}\right\} z_{r}^{s} d x^{t}\right)\right)+\sum_{k=2}^{n} d z_{k}^{2} \wedge d z_{r}^{k} \\
& =\sum_{j=1}^{n} d z_{\imath}^{j} \wedge d z_{r}^{j}+d\left\{\begin{array}{c}
i \\
r t
\end{array}\right\} \wedge d x^{t}+\sum_{k=2}^{n} d z_{k}^{i} \wedge d z_{r}^{k} \\
& =d z_{\imath}^{1} \wedge d z_{r}^{1}+\partial_{s}\left\{\begin{array}{c}
i \\
r t
\end{array}\right\} d x^{s} \wedge d x^{t} \\
& =\zeta^{i} \wedge \zeta^{\bar{r}}+\partial_{s}\left\{\begin{array}{c}
i \\
r t
\end{array}\right\} d x^{s} \wedge d x^{t},
\end{aligned}
$$


i. e. $T_{r s t}^{\prime i}=R_{r s t}^{i}, T_{r j k}^{\prime i}=\delta_{j}^{i} \delta_{r k}-\delta_{k}^{i} \delta_{r j}$ and $T_{r \beta \gamma}^{\prime i}=0$ otherwise. q.e.d.

Since $\left(Q, \chi^{\prime}\right)$ is an $H_{0}$-reduction desired in [M1, Proposition 5.1], we can apply [M1, Proposition 5.2] to it. Namely, as in the existence proof of the connection $(Q, \chi)$ there, put

$$
\begin{aligned}
& A_{0 j}^{1}=-\frac{1}{n-2} \sum_{i=2}^{n} T_{j i}^{\prime \bar{i}}=\frac{1}{n-2} \sum_{\imath} R_{1 \imath j}^{\imath}=\frac{1}{n-2} R_{1 \jmath}, \\
& A_{0 j}^{0}=A_{1 j}^{0}=A_{0 j}^{1}=A_{0 j}^{0}=A_{1 j}^{0}=A_{k \imath}^{2}=A_{i \bar{i}}^{k}=A_{\imath j}^{k}=A_{\imath j}^{k}=0,
\end{aligned}
$$

for $2 \leqq i, j, k \leqq n$, where we use the Ricci curvature tensor $R_{j k}=\sum_{i=1}^{n} R_{j i k}^{2}$ of $M$ at $p$. The scalar curvature tensor of $M$ is denoted by $R=\sum_{j=1}^{n} R_{j j}$. In the following, we also use the notation $\widetilde{R}_{j k}=\sum_{i=2}^{n} R_{j i k}^{\imath}, 1 \leqq j, k \leqq n$, and $\tilde{R}=\sum_{i=2}^{n} \widetilde{R}_{j j}$. Immediately, we have

$$
\begin{aligned}
& R_{1 \jmath}=\widetilde{R}_{1 \jmath}, \quad 1 \leqq j \leqq n, \\
& R_{\jmath k}=\widetilde{R}_{j k}+R_{j 1 k}^{1}, \quad 2 \leqq j, k \leqq n, \\
& R=\sum_{i=2}^{n} R_{i i}+R_{11}=\widetilde{R}+2 R_{11} .
\end{aligned}
$$

Let $\Psi^{\prime \prime}=(1 / 2) T^{\prime \prime} \zeta \wedge \zeta$ be the curvature of the connection $\chi^{\prime \prime}$ defined by

$$
\chi^{\prime \prime 1}{ }_{0}=\chi_{0}^{\prime 1}+\sum_{j=2}^{n} A_{0 j}^{\prime} \zeta^{j}
$$

and $\chi^{\prime \prime}=\chi^{\prime}$ for other indices. Then we have $\Psi^{\prime \prime}=\Psi^{\prime}$ except for

$$
\Psi_{0}^{\prime \prime 1}-\Psi_{0}^{\prime 1}=d\left(\sum_{j=2}^{n} A_{0 j}^{1} \zeta^{j}\right)-2 \chi_{0}^{\prime 0} \wedge A_{0 j}^{1} \zeta^{j}
$$

Note that $T^{\prime \prime}{ }_{0 i}^{1}=-\partial_{i} A_{0 \imath}^{1}$. Now, putting

where

$$
\chi_{\beta}^{\alpha}=\chi_{\beta}^{\prime \prime}{ }_{\beta}^{\alpha}+A_{\beta 1}^{\alpha} \zeta^{1},
$$

$$
\begin{aligned}
& A_{01}^{0}=-\frac{1}{2(n-1)} \Sigma\left(T^{\prime \prime}{ }_{12}^{i}-T^{\prime \prime 0}{ }_{0 i \bar{i}}\right)=0 \text {, } \\
& A_{11}^{0}=-\frac{1}{2(n-1)}\left(\sum_{i=2}^{n} T_{12}^{\prime \prime 2}-T^{\prime \prime 0}{ }_{0 i \bar{i}}\right)=-\frac{1}{2} \text {, } \\
& A_{01}^{1}=-\frac{1}{2(n-1)} \sum_{i=2}^{n}\left(T^{\prime \prime i}{ }_{1 i}-T^{\prime \prime 1}{ }_{0 i \bar{i}}\right)=\frac{1}{2(n-1)}\left(R_{11}-\sum_{i} \partial_{\bar{i}} A_{0 i}^{1}\right) \text {, } \\
& A_{j 1}^{2}=-\frac{1}{n+3}\left(T^{\prime \prime}{ }_{1 j}-T^{\prime \prime}{ }_{1 \imath}+T^{\prime \prime}{ }_{1 j}^{i}-T^{\prime \prime}{ }_{1 i}-\sum_{k} T^{\prime \prime}{ }_{j k \bar{k}}\right)=0,
\end{aligned}
$$

we obtain the desired $(Q, \chi)$. Here, note that the curvatures given in Proposi- 
tion 2.2 and so these coefficients are given pointwise. Thus, to get $A_{01}^{1}$ explicitely, we must compute

$$
\partial_{j} A_{0 \imath}^{1}=-\frac{1}{n-2} \sum_{m=2}^{n}\left(\partial_{j} T_{\imath m}^{\prime m}\right)
$$

From

$$
\begin{aligned}
\Psi^{\prime \bar{m}} & =d\left(g_{r k} z_{m}^{k}\left(d z_{1}^{r}+\left\{\begin{array}{c}
r \\
s t
\end{array}\right\} z_{1}^{s} d x^{t}\right)\right)+\sum_{r=2}^{n} \chi_{r}^{\prime m} \wedge \zeta^{\bar{r}} \\
& \equiv \partial_{u}\left(g_{r k} z_{m}^{k}\left\{\begin{array}{c}
r \\
s t
\end{array}\right\} z_{1}^{s}\right) z_{\imath}^{u} z_{v}^{t} \zeta^{i} \wedge \zeta^{v} \quad\left(\bmod \zeta^{\bar{r}} \wedge \zeta^{r}\right),
\end{aligned}
$$

we have for $j, m \geqq 2$,

$$
\begin{aligned}
\partial_{j} \Psi^{\prime m} \equiv & \left\{\partial_{j} \partial_{u}\left(g_{r k}\left\{\begin{array}{c}
r \\
s t
\end{array}\right\} z_{m}^{k} z_{1}^{s}\right) z_{\imath}^{u} z_{v}^{t}+\partial_{u}\left(g_{r k}\left\{\begin{array}{c}
r \\
s t
\end{array}\right\} z_{m}^{k} z_{1}^{s}\right) \partial_{j}\left(z_{\imath}^{u} z_{v}^{t}\right)\right\} \zeta^{i} \wedge \zeta^{v} \\
= & \left\{\partial_{u}\left(g_{r k}\left\{\begin{array}{c}
r \\
s t
\end{array}\right\} \partial_{j}\left(z_{m}^{k} z_{1}^{s}\right)\right) \delta_{i}^{u} \delta_{v}^{t}\right. \\
& +\left(\partial_{u}\left\{\begin{array}{l}
m \\
1 t
\end{array}\right\}\right)\left\{\left(\delta_{j}^{u} \delta_{i 1}-\delta_{j i} \delta_{1}^{u}\right) \delta_{v}^{t}+\delta_{i}^{u}\left(\delta_{j}^{t} \delta_{v 1}-\delta_{j v} \delta_{1}^{t}\right)\right\} \zeta^{i} \wedge \zeta^{v} \\
= & \left\{\partial_{u}\left\{\begin{array}{l}
k \\
s t
\end{array}\right\}\left(-\delta_{\jmath m} \delta_{1}^{k} \delta_{1}^{s}+\delta_{m}^{k} \delta_{j}^{s}\right) \delta_{i}^{u} \delta_{v}^{t}+\partial_{j}\left\{\begin{array}{l}
m \\
1 v
\end{array}\right\} \delta_{i 1}-\partial_{1}\left\{\begin{array}{l}
m \\
1 v
\end{array}\right\} \delta_{j i}\right. \\
& \left.+\partial_{i}\left\{\begin{array}{l}
m \\
1 j
\end{array}\right\} \delta_{v 1}-\partial_{i}\left\{\begin{array}{l}
m \\
11
\end{array}\right\} \delta_{j v}\right\} \zeta^{i} \wedge \zeta^{v} \\
= & \left\{-\partial_{i}\left\{\begin{array}{l}
1 \\
1 v
\end{array}\right\} \delta_{j m}+\partial_{i}\left\{\begin{array}{l}
m \\
j v
\end{array}\right\}+\partial_{j}\left\{\begin{array}{l}
m \\
1 v
\end{array}\right\} \delta_{i 1}-\partial_{1}\left\{\begin{array}{l}
m \\
1 v
\end{array}\right\} \delta_{j i}+\partial_{i}\left\{\begin{array}{l}
m \\
1 j
\end{array}\right\} \delta_{v 1}\right. \\
& \left.-\partial_{i}\left\{\begin{array}{l}
m \\
11
\end{array}\right\} \delta_{j v}\right\} \zeta^{i} \wedge \zeta^{v} .
\end{aligned}
$$

Thus we obtain

$$
\partial_{j} T_{\imath v}^{\prime \bar{m}}=R_{j i v}^{m}+R_{1 j v}^{m} \delta_{i 1}-R_{11 v}^{m} \delta_{j i}+R_{1 \imath j}^{m} \delta_{v 1}-R_{1 i 1}^{m} \delta_{j v},
$$

for $1 \leqq i, v \leqq n, 2 \leqq j, m \leqq n$, and it follows for $2 \leqq i, j \leqq n$,

$$
\begin{aligned}
\partial_{j} A_{0 i}^{1} & =-\frac{1}{n-2}\left(-\widetilde{R}_{j i}+R_{11} \delta_{j i}-R_{1 i 1}^{\jmath}\right) \\
& =\frac{1}{n-2}\left(R_{\imath j}-R_{11} \delta_{j}^{i}\right)
\end{aligned}
$$

using (2.12). Finally we have 


$$
\begin{aligned}
A_{01}^{1} & =\frac{1}{2(n-1)}\left(R_{11}-\sum_{i=2}^{n} \partial_{\bar{i}} A_{0 \imath}^{1}\right) \\
& =\frac{1}{2(n-1)}\left\{R_{11}-\frac{1}{n-2}\left(\sum_{\imath=2}^{n} R_{i i}-(n-1) R_{11}\right)\right\} \\
& =\frac{1}{n-2} R_{11}-\frac{1}{2(n-1)(n-2)} R .
\end{aligned}
$$

Thus, the Cartan connection $(Q, \chi)$ of type $H / H_{0}$ defined by

$$
\begin{aligned}
& \chi_{-}=\chi_{-}^{\prime}, \quad \chi_{r}^{i}=\chi_{r}^{\prime i}, \quad \chi_{0}^{0}=\chi_{0}^{\prime 0}, \\
& \chi_{1}^{0}=\chi_{1}^{\prime 0}+A_{11}^{0} \zeta^{1}, \quad \chi_{0}^{1}=\chi_{0}^{\prime 1}+\sum_{j=2}^{n} A_{0 j}^{1} \zeta^{j}+A_{01}^{1} \zeta^{1},
\end{aligned}
$$

is normal (i.e. $T^{-1}=\partial^{*} T^{0}=\left(\partial^{*} T^{1}\right)\left(e_{1}\right)=0$, where $\Psi=(1 / 2) T \zeta \wedge \zeta$ is its curvature) [M1, Proposition 5.2].

Proposition 2.4. The curvature $\Psi=(1 / 2) T \zeta \wedge \zeta$ of $(Q, \chi)$ is given by

$T_{-2}=0$,

$T_{1 j}^{i}=\frac{1}{2} \delta_{\jmath}^{2}, \quad T_{\beta r}^{\imath}=0$ otherwise,

$T_{1 j}^{\bar{i}}=R_{11 j}^{\imath}+\delta_{j}^{\imath}\left\{\frac{1}{n-2} R_{11}-\frac{R}{2(n-1)(n-2)}\right\}$,

$T_{1 j}^{\bar{i}}=0$,

$T_{j k}^{\bar{i}}=R_{1 j k}^{2}-\frac{1}{n-2}\left(R_{1 k} \delta_{j}^{2}-R_{1 j} \delta_{k}^{i}\right)$,

$T_{i j}^{i_{j \gamma}}=0$,

$T_{j k m}^{i}=R_{j k m}^{\imath}, \quad T_{j \bar{k} \bar{m}}^{i}=\delta_{k}^{i} \delta_{\jmath m}-\delta_{m}^{\imath} \delta_{j k}, \quad T_{j \beta \gamma}^{i}=0$ otherwise,

$T_{01 i}^{0}=-\frac{1}{2(n-2)} R_{1 \imath}, \quad T_{0 \beta \gamma}^{0}=0$ otherwise,

$T_{1 i \bar{\imath}}^{0}=\frac{1}{2}, \quad T_{1 \beta \gamma}^{0}=0$ otherwise,

$T_{01 \jmath}^{1}=\frac{1}{n-2} \partial_{1} R_{1 j}-\frac{1}{n-2} \partial_{j} R_{11}+\frac{1}{2(n-1)(n-2)} \partial_{j} R$,

$T_{01 j}^{1}=-\partial_{j} A_{01}^{1}+\frac{1}{n-2} R_{1 \jmath}$,

$T_{0 \imath \jmath}^{1}=\frac{1}{n-2}\left(\partial_{i} R_{1 j}-\partial_{j} R_{1 \imath}\right)$, 


$$
\begin{aligned}
& T_{0 i j}^{1}=\frac{1}{n-2} R_{\imath j}-\frac{R}{2(n-1)(n-2)} \delta_{j}^{\imath} \\
& T_{0 i \bar{j} j}^{1}=0,
\end{aligned}
$$

where $2 \leqq i, j, k \leqq n$ and $\beta, \gamma \in\{1, \cdots, n, \overline{2}, \cdots, \bar{n}\}$.

Proof. We use the relation between $T$ and $T^{\prime}$ obtained in [M1, §5]. The first one is obvious. Next, we have

$$
\begin{aligned}
T_{1 j}^{i} & =T_{1 j}^{\prime i}+A_{11}^{0} \delta_{j}^{i}=\frac{1}{2} \delta_{j}^{i}, \\
T_{1 j}^{\bar{i}} & =T_{1 j}^{\prime i}+A_{01}^{1} \delta_{j}^{i} \\
& =R_{11 j}^{2}+\delta_{j}^{i}\left(\frac{1}{n-2} R_{11}-\frac{R}{2(n-1)(n-2)}\right) \\
T_{j k}^{\bar{i}} & =T_{j k}^{\prime \bar{i}}-A_{0 k}^{1} \delta_{j}^{i}+A_{0 j}^{1} \delta_{k}^{i} \\
& =R_{1 j k}^{2}-\frac{1}{n-2}\left(R_{1 k} \delta_{j}^{i}-R_{1 j} \delta_{k}^{i}\right), \quad 2 \leqq j, k \leqq n, \\
T_{j \gamma}^{\bar{i}} & =T_{j r}^{\prime \bar{i}}=0, \\
T_{r \beta \gamma}^{i} & =T_{r \beta r}^{\prime i}=R_{r s t}^{2} \delta_{\beta}^{s} \delta_{r}^{t}+\delta_{\beta}^{\bar{i}} \delta_{\bar{r} \gamma}-\delta_{r}^{\bar{i}} \delta_{\bar{r} \beta}, \quad 2 \leqq i, r, s, t \leqq n .
\end{aligned}
$$

Now, from

$$
\begin{aligned}
\Psi_{0}^{0}-\Psi_{0}^{\prime 0} & =\chi_{1}^{0} \wedge \chi_{0}^{1}-\chi_{1}^{\prime 0} \wedge \chi_{0}^{\prime 1} \\
& =\left(\chi_{1}^{\prime 0}+A_{11}^{0} \zeta^{1}\right) \wedge\left(\chi_{0}^{\prime 1}+A_{0 j}^{1} \zeta^{j}+A_{01}^{1} \zeta^{1}\right)-\chi_{1}^{\prime 0} \wedge \chi_{0}^{\prime 1} \\
& A_{11}^{0} \zeta^{1} \wedge\left(\sum_{j=2}^{n} A_{0 j}^{1} \zeta^{j}+A_{01}^{1} \zeta^{1}\right)
\end{aligned}
$$

we get

$$
T_{01 \imath}^{0}=T_{01 i}^{\prime 0}+A_{11}^{0} A_{02}^{1}=-\frac{1}{2(n-2)} R_{1 \imath}, \quad T_{0 \beta \gamma}^{0}=T_{0 \beta \gamma}^{\prime 0}=0, \quad \text { otherwise. }
$$

Similarly, from

$$
\Psi_{1}^{0}-\Psi_{1}^{\prime 0}=d\left(A_{11}^{0} \zeta^{1}\right)=-\frac{1}{2} d \zeta^{1}=\frac{1}{2} \sum_{i=2}^{n} \zeta^{i} \wedge \zeta^{\bar{i}}
$$

we obtain

$$
T_{1 i \bar{i}}^{0}=\frac{1}{2}, \quad T_{1 \beta \gamma}^{0}=0, \quad \text { otherwise. }
$$

Then, from

$$
\Psi_{0}^{1}-\Psi_{0}^{\prime 1}=d\left(\sum_{j=2}^{n} A_{0 j}^{1} \zeta^{j}+A_{01}^{1} \zeta^{1}\right)=\sum_{j=2}^{n} d A_{0 j}^{1} \wedge \zeta^{j}+d A_{01}^{1} \wedge \zeta^{1}+\sum_{j=2}^{n} A_{0 j}^{1} d \zeta^{j}+A_{01}^{1} d \zeta^{1},
$$

to obtain $T_{01 j}^{1}=T_{{ }_{01 j}}^{\prime 1}+\partial_{1} A_{0 j}^{1}-\partial_{j} A_{01}^{1}$, we compute 


$$
\partial_{i} A_{0 \jmath}^{1}=-\frac{1}{n-2} \partial_{i}\left(\sum_{m} T_{\jmath m}^{\prime m}\right), \quad 2 \leqq i, j \leqq n .
$$

Here we use (2.9) and get

$$
\begin{aligned}
\partial_{i} \Psi^{\prime \bar{m}} & \equiv \partial_{i}\left(\partial_{u}\left(g_{r_{k}} z_{m}^{k}\left\{\begin{array}{c}
r \\
s t
\end{array}\right\} z_{1}^{s}\right) z_{j}^{u} z_{v}^{t}\right) \zeta^{j} \wedge \zeta^{v} \quad\left(\bmod \zeta^{\bar{r}} \wedge \zeta^{r}\right) \\
& =\partial_{i} \partial_{u}\left(g_{r k} z_{m}^{k}\left\{\begin{array}{c}
r \\
s t
\end{array}\right\}\right) \delta_{1}^{s} \delta_{j}^{u} \delta_{v}^{t} \zeta^{j} \wedge \zeta^{v} \\
& =\partial_{i} \partial_{j}\left\{\begin{array}{l}
m \\
1 v
\end{array}\right\} \zeta^{j} \wedge \zeta^{v},
\end{aligned}
$$

and so

$$
\partial_{i} A_{0 j}^{1}=-\frac{1}{n-2} \partial_{i} \sum_{m} R_{1 \jmath m}^{m}=\frac{1}{n-2} \partial_{i} R_{1 \jmath}, \quad 1 \leqq i \leqq n \quad \text { and } \quad 2 \leqq j \leqq n .
$$

Similarly, we get

$$
\partial_{j} A_{01}^{1}=\frac{1}{n-2} \partial_{j} R_{11}-\frac{1}{2(n-1)(n-2)} \partial_{j} R .
$$

Therefore, we have

$$
\begin{aligned}
& T_{01 j}^{1}=T_{01 j}^{\prime 1}+\partial_{1} A_{0 j}^{1}-\partial_{j} A_{01}^{1} \\
& =\frac{1}{n-2} \partial_{1} R_{1 j}-\frac{1}{n-2} \partial_{j} R_{11}+\frac{1}{2(n-1)(n-2)} \partial_{j} R, \\
& T_{0 \imath j}^{1}=T_{0 \imath j}^{\prime 1}+\partial_{i} A_{0 j}^{1}-\partial_{\jmath} A_{0 \imath}^{1} \\
& =\frac{1}{n-2}\left(\partial_{i} R_{1 j}-\partial_{j} R_{12}\right), \\
& T_{0 \bar{\imath}_{j}}^{1}=T^{\prime 1}{ }_{0 i j}+\partial_{i} A_{0 j}^{1}+A_{01}^{1} \delta_{j}^{i} \\
& =\frac{1}{n-2}\left(R_{\imath j}-R_{11} \delta_{j}^{i}\right)+\delta_{j}^{i}\left\{\frac{1}{n-2} R_{11}-\frac{R}{2(n-1)(n-2)}\right\} \\
& =\frac{1}{n-2} R_{\imath j}-\frac{R}{2(n-1)(n-2)} \delta_{j}^{i}, \\
& T_{0 i \bar{j}}^{1}=T^{\prime 1}{ }_{0 i \bar{j}}=0 \text {. }
\end{aligned}
$$

Now, we have

$$
T_{01 j}^{1}=T^{\prime 1}{ }_{01 j}-\partial_{j} A_{01}^{1}+A_{0 j}^{1}=-\partial_{j} A_{01}^{1}+\frac{1}{n-2} R_{1 j},
$$

where $\partial_{j} A_{01}^{1}$ is given later (Lemma 4.3).

q.e.d. 


\section{§ 3. A Cartan connection.}

Using the method of the proof of [M1, Proposition 5.3], we construct Tanaka connection on $P=Q \times{ }_{H_{0}} G^{\prime}$ from the normal Cartan connection $(Q, \chi)$ constructed in $\S 2$.

First of all, for later use, recall the structure equation $[\mathrm{M} 1,(3.1)]$, of a Cartan connection $(P, \omega)$ of the $G / G^{\prime}$, where $\theta=\omega_{-2}+\omega_{-1}$ is the basic form, and $\Omega$ is the curvature form:

$$
\begin{aligned}
& d \theta^{1}=-\left(\omega_{0}^{0}+\omega_{1}^{1}\right) \wedge \theta^{1}-\sum_{i=2}^{n} \theta^{\imath} \wedge \theta^{i}+\Omega^{1}, \\
& d \theta^{2}=\omega_{\bar{i}} \wedge \theta^{1}-\sum_{j=2}^{n} \omega_{j}^{2} \wedge \theta^{\jmath}+\theta^{\imath} \wedge \omega_{0}^{0}+\theta^{i} \wedge \omega_{1}^{0}+\Omega^{\imath}, \\
& d \theta^{\bar{i}}=-\omega_{i} \wedge \theta^{1}-\sum_{j=2}^{n} \omega_{j}^{2} \wedge \theta^{j}+\theta^{2} \wedge \omega_{0}^{1}+\theta^{\bar{i}} \wedge \omega_{1}^{1}+\Omega^{\bar{i}}, \\
& d \omega_{0}^{0}=-\omega_{1}^{0} \wedge \omega_{0}^{1}-\sum_{i=2}^{n} \theta^{\imath} \wedge \omega_{i}-\theta^{1} \wedge \omega_{1}+\Omega_{0}^{0}, \\
& d \omega_{1}^{0}=-\left(\omega_{0}^{0}-\omega_{1}^{1}\right) \wedge \omega_{1}^{0}-\sum_{\imath=2}^{n} \theta^{\imath} \wedge \omega_{i}+\Omega_{1}^{0}, \\
& d \omega_{0}^{1}=\left(\omega_{0}^{0}-\omega_{1}^{1}\right) \wedge \omega_{0}^{1}-\sum_{i=2}^{n} \theta^{\bar{i}} \wedge \omega_{i}+\Omega_{0}^{1}, \\
& d \omega_{1}^{1}=-\omega_{0}^{1} \wedge \omega_{1}^{0}-\sum_{i=2}^{n} \theta^{\bar{i}} \wedge \omega_{\bar{i}}-\theta^{1} \wedge \omega_{1}+\Omega_{1}^{1}, \\
& d \omega_{j}^{i}=-\omega_{i} \wedge \theta^{\jmath}-\omega_{i} \wedge \theta^{j}-\sum_{k=2}^{n} \omega_{k}^{2} \wedge \omega_{j}^{k}-\theta^{2} \wedge \omega_{j}-\theta^{\bar{\imath}} \wedge \omega_{j}+\Omega_{j}^{i}, \\
& d \omega_{i}=-\omega_{i} \wedge \omega_{0}^{0}-\omega_{i} \wedge \omega_{0}^{1}-\theta^{\bar{i}} \wedge \omega_{1}+\sum_{j=2}^{n} \omega_{i}^{\jmath} \wedge \omega_{j}+\Omega_{\imath}, \\
& d \omega_{\bar{i}}=-\omega_{i} \wedge \omega_{1}^{0}-\omega_{\bar{i}} \wedge \omega_{1}^{1}+\theta^{i} \wedge \omega_{1}+\sum_{j=2}^{n} \omega_{i}^{\jmath} \wedge \omega_{j}+\Omega_{\bar{i}}, \\
& d \omega_{1}=\left(\omega_{0}^{0}+\omega_{1}^{1}\right) \wedge \omega_{1}+\sum_{i=2}^{n} \omega_{i} \wedge \omega_{\bar{i}}+\Omega_{1} .
\end{aligned}
$$

Now, let $\omega^{\prime}$ be the Cartan connection on $P$ naturally extended from $\chi$. Namely, let $\left(x^{2}, z_{\jmath}^{2}, s_{b}^{a}, s_{\imath}, s_{i}^{i}, s_{1}\right)$ be a local coordinate on $P$ where $\left(x^{i}, z_{j}^{i}\right)$ is the local coordinate of $P_{\boldsymbol{g}}$ around a point $u_{0} \in P_{\boldsymbol{g}}$ chosen in $\S 2,\left(s_{b}^{a}\right) \in G L(2, \boldsymbol{R}), 0 \leqq a$, $b \leqq 1, s_{i}=s_{i}^{n+1}, s_{i}=s_{i}^{n+2}, 2 \leqq i \leqq n$ and $s_{1}=s_{0}^{n+2}$. As in $\S 2$, we define $\omega^{\prime}$ by $\omega_{-2}^{\prime}+$ $\omega_{-1}^{\prime}=\chi_{-2}+\chi_{-1}, \omega_{b}^{\prime a}=\chi_{b}^{a}, 0 \leqq a, b \leqq 1, \omega_{i}^{\prime}=d s_{i}, \omega_{i}^{\prime}=d s_{i}^{-}$and $\omega_{1}^{\prime}=d s_{1}$ at $u \in Q$ (note that $\left.\left.s_{b}^{a}\right|_{Q}=h_{b}^{a}\right)$, and then extend it to $P$ by $R_{a}^{*} \omega^{\prime}=A d\left(a^{-1}\right) \omega^{\prime}$ where $a \in G^{\prime}$. Obviously, $\left(P, \omega^{\prime}\right)$ is a Cartan connection of type $G / G^{\prime}$ with basic form $\theta=\omega_{-2}^{\prime}$ $+\omega_{-1}^{\prime}$. 
Proposition 3.1. The curvature $\Omega^{\prime}=(1 / 2) K^{\prime} \theta \wedge \theta$ of $\omega^{\prime}$ at $u_{0}$ satisfies

$$
\iota^{*} K^{\prime}=T
$$

where $\iota: Q \rightarrow P$ is the inclusion map and $T$ is given in Proposition 2.4,

and

$$
K^{\prime 1}{ }_{11}=\frac{1}{2(n-2)} R_{1 \jmath}, \quad K_{1 \beta \gamma}^{\prime 1}=0, \text { otherwise, }
$$

$$
K_{i \beta \gamma}^{\prime}=K_{i \beta \gamma}^{\prime}=K_{1 \beta \gamma}^{\prime}=0 \text {. }
$$

Proof. The non-trivial case is $\Omega^{\prime 1}$. From the structure equation (3.1), we get

$$
\begin{aligned}
\Omega_{1}^{\prime 1} & =d \omega_{1}^{\prime 1}+\left(\chi^{\prime \prime}{ }_{0}+\sum_{j=2}^{n} A_{0 j}^{1} \theta^{\jmath}+A_{01}^{1} \theta^{1}\right) \wedge\left(\chi^{\prime 0}{ }_{1}+A_{11}^{0} \theta^{1}\right)+\sum_{i=2}^{n} \theta^{i} \wedge \omega_{i}+\theta^{1} \wedge \omega_{1} \\
& =\sum_{j=2}^{n} A_{0 j}^{1} A_{11}^{0} \theta^{\jmath} \wedge \theta^{1},
\end{aligned}
$$

and so

$$
K_{1 j 1}^{\prime 1}=-\frac{1}{2(n-2)} R_{1 \jmath}, \quad K_{1 \beta \gamma}^{\prime 1}=0 \text { otherwise. } \quad \text { q.e.d. }
$$

Now, we construct a Cartan connection $\left(P, \omega^{\prime \prime}\right)$ as in [M1]. To obtain $A_{23}$ for $i \neq j$ in [M1], by Proposition 3.1, 2.4 and (2.12), we get

$$
\begin{aligned}
-K_{1 j}^{\prime \bar{i}}+K_{0 \imath j}^{\prime 0}+K^{\prime 1}{ }_{0 i j}-\sum_{k=2}^{n} K_{k k j}^{\prime \imath} & =R_{1 j 1}^{\imath}+\frac{1}{n-2} R_{\imath j}-\sum_{k=2}^{n} R_{k k \jmath}^{\imath} \\
& =\frac{n-1}{n-2} R_{\imath \jmath},
\end{aligned}
$$

so that noting $A_{\imath \jmath}=A_{j i}$, we have

$$
A_{\imath j}=-\frac{1}{n-2} R_{\imath \jmath}
$$

For $i=j$, we get

$$
\begin{aligned}
-K_{1 i}^{\prime i}+K_{0 i i}^{\prime 0}+K_{0 i i}^{\prime 1}-\sum_{k=2}^{n} K_{k k i}^{\prime i}= & R_{1 i 1}^{2}-\frac{1}{n-2} R_{11}+\frac{R}{2(n-1)(n-2)} \\
& +\frac{1}{n-2} R_{i i}-\frac{R}{2(n-1)(n-2)}+\widetilde{R}_{i i} \\
= & \frac{n-1}{n-2} R_{i i}-\frac{1}{n-2} R_{11},
\end{aligned}
$$

and the summation over $2 \leqq i \leqq n$ gives

$$
\sum_{i=2}^{n}\left(-K_{1 i}^{\prime \bar{i}}+K^{\prime 0}{ }_{0 i i}-\sum_{k=2}^{n} K_{k k i}^{\prime i}+K_{0 i \imath}^{\prime 1}\right)=\frac{n-1}{n-2}\left(R-R_{11}\right)-\frac{n-1}{n-2} R_{11}
$$




$$
=\frac{n-1}{n-2}\left(R-2 R_{11}\right)
$$

Therefore, we get

$$
\begin{aligned}
A_{i i} & =-\frac{1}{n-2} R_{i i}+\frac{1}{(n-1)(n-2)} R_{11}+\frac{1}{2(n-1)(n-2)}\left(R-2 R_{11}\right) \\
& =-\frac{1}{n-2} R_{i i}+\frac{R}{2(n-1)(n-2)}
\end{aligned}
$$

or,

$$
A_{\imath \jmath}=-\frac{1}{n-2} R_{\imath j}+\frac{R}{2(n-1)(n-2)} \delta_{\jmath}^{2} .
$$

The Left hand sides of $(7)^{\prime}$ and $(8)^{\prime}$ vanish so that we get

$$
A_{i \jmath}=A_{i j}=0 \text {. }
$$

As for $(9)^{\prime}$, since we have

$$
\begin{aligned}
& {K^{\prime}}_{1 j}^{i}+K^{\prime 0}{ }_{12 j}+K^{\prime 1}{ }_{1 \bar{j} j}-\sum_{k=2}^{n} K_{k \bar{k} j}^{\prime \imath}=\frac{1}{2} \delta_{j}^{i}+\frac{1}{2} \delta_{j}^{i}-\sum_{k=2}^{n}\left(\delta_{k}^{i} \delta_{k j}-\delta_{j}^{i}\right) \\
& =\delta_{j}^{i}-\delta_{j}^{i}+(n-1) \delta_{j}^{i} \\
& =(n-1) \delta_{j}^{i} \text {, }
\end{aligned}
$$

we get

$$
A_{\bar{i} j}=-\frac{1}{2} \delta_{j}^{i}
$$

Therefore, $\omega^{\prime \prime}$ is given by

$$
\omega_{p}^{\prime \prime}=\omega_{p}^{\prime}, \quad p \leqq 0, \quad \omega_{i}^{\prime \prime}=\omega_{i}^{\prime}+\sum_{j=2}^{n} A_{\imath j} \theta^{\jmath}, \quad \omega_{i}^{\prime \prime}=\omega_{i}^{\prime}-\frac{1}{2} \theta^{\bar{i}}, \quad \omega_{1}^{\prime \prime}=\omega_{1}^{\prime} .
$$

Proposition 3.2. The curvature $\Omega^{\prime \prime}=\frac{1}{2} K^{\prime \prime} \theta \wedge \theta$ of $\omega^{\prime \prime}$ is given at $u_{0} \in P_{g}$ by,

$$
\begin{aligned}
& K_{-2}^{\prime \prime}=0 \text {, } \\
& K_{\beta r}^{\prime \prime i}=0 \text {, } \\
& K_{1 j}^{\prime \prime}=R_{11 j}^{\imath}+\frac{1}{n-2} R_{\imath j}+\frac{1}{n-2} R_{11} \delta_{j}^{i}-\frac{R}{(n-1)(n-2)} \delta_{j}^{\imath}, \\
& K_{1 j}^{\prime \prime \bar{i}}=K_{1 j}^{\prime \bar{i}}=0 \text {, } \\
& K_{j k}^{\prime \prime \bar{i}}=R_{1 j k}^{\imath}-\frac{1}{n-2}\left(R_{1 k} \delta_{\jmath}^{\imath}-R_{1 j} \delta_{k}^{j}\right), \quad K_{\beta \gamma}^{\prime \prime \bar{i}}=0 \text { otherwise, } \\
& K^{\prime \prime i}{ }_{j k 1}^{i}=R_{j k 1}^{\imath} \text {, }
\end{aligned}
$$




$$
\begin{aligned}
& K^{\prime \prime}{ }_{j k m}=R_{j k m}^{\imath}-\frac{1}{n-2}\left(R_{i k} \delta_{m}^{\jmath}-R_{\imath m} \delta_{k}^{\jmath}+R_{\jmath m} \delta_{k}^{i}-R_{j k} \delta_{m}^{2}\right) \\
& +\frac{R}{(n-1)(n-2)}\left(\delta_{k}^{i} \delta_{\jmath m}-\delta_{m}^{2} \delta_{j k}\right) \\
& K^{\prime \prime i}{ }_{j \bar{k} \gamma}=K^{\prime i}{ }_{j \bar{k} \gamma}=0 \text {, } \\
& K^{\prime \prime 0}{ }_{01 \jmath}=-\frac{1}{2(n-2)} R_{1 \jmath} \text {, } \\
& K^{\prime \prime 0}{ }_{0 \imath \jmath}=0 \text {, } \\
& K^{\prime \prime{ }_{0 \bar{\gamma}}}=K_{0 \bar{i} \gamma}^{\prime 0}=0 \text {, } \\
& K^{\prime \prime 1 \beta \gamma}=K_{1 \beta \gamma}^{\prime 0}=0 \text {, } \\
& K^{\prime \prime 11}=\frac{1}{n-2} \partial_{1} R_{1 j}-\frac{1}{n-2} \partial_{j} R_{11}+\frac{1}{2(n-1)(n-2)} \partial_{j} R \text {, } \\
& K^{\prime \prime 1}{ }_{01 j}^{1}=-\partial_{j} A_{01}^{1}+\frac{1}{n-2} R_{1 j} \text {, } \\
& K^{\prime \prime 1}{ }_{0 \imath \jmath}=\frac{1}{n-2}\left(\partial_{i} R_{1 j}-\partial_{j} R_{1 \imath}\right), \quad K_{0 B \gamma}^{\prime 11}=0 \text { otherwise, } \\
& K^{\prime \prime 1}{ }_{11}^{1}=\frac{1}{2(n-2)} R_{1 \jmath}, \quad K^{\prime \prime 1}{ }_{1 \beta \gamma}=K^{\prime 1}{ }_{1 \beta \gamma}=0, \text { otherwise, } \\
& K_{i 1 j}^{\prime \prime}=\partial_{1} A_{\imath \jmath} \text {, } \\
& K_{i 1 j}^{\prime \prime}=A_{\imath j}+\frac{1}{2} A_{01}^{1} \delta_{j}^{i}, \\
& K_{\imath j k}^{\prime \prime}=-\partial_{k} A_{\imath j}+\partial_{\jmath} A_{i k}, \\
& K_{i j \bar{k}}^{\prime \prime}=-\partial_{\bar{k}} A_{\imath j}+\frac{1}{2(n-2)} R_{1 j} \delta_{k}^{2}, \\
& K_{\bar{i} 1 \jmath}^{\prime \prime}=-\frac{1}{2} R_{11 j}^{\imath}+\frac{1}{2} A_{\imath \jmath} \text {, } \\
& K_{\bar{i}_{j k}}^{\prime \prime}=-\frac{1}{2} R_{1 j k}^{2}, \quad K_{\frac{i}{\beta \gamma}}^{\prime \prime}=0 \text { otherwise, } \\
& K_{1 \bar{\imath} \jmath}^{\prime \prime}=-\frac{1}{2} A_{\imath \jmath}, \\
& K_{1 \beta \gamma}^{\prime \prime}=0 \text { otherwise. }
\end{aligned}
$$

Proof. We can compute $\Omega^{\prime \prime}-\Omega^{\prime}$ by using (3.1) as follows:

$$
\Omega^{\prime \prime 1}-\Omega^{\prime 1}=0 \longleftrightarrow K_{-2}^{\prime \prime}=0,
$$




$$
\begin{aligned}
& \Omega^{\prime \prime \imath}-\Omega^{\prime \imath}=\frac{1}{2} \theta^{i} \wedge \theta^{1} \\
& \longleftrightarrow K_{1 j}^{\prime \prime i}=K_{1 j}^{\prime i}-\frac{1}{2} \delta_{j}^{i}=0 \\
& K_{\beta \gamma}^{\prime \prime i}=K_{\beta \gamma}^{\prime i}=0 \quad \text { otherwise, } \\
& \Omega^{\prime \prime \bar{\imath}}-\Omega^{\prime i}=A_{2 j} \theta^{\jmath} \wedge \theta^{1} \\
& \longleftrightarrow K_{1 \jmath}^{\prime \prime \bar{i}}=K_{1 j}^{\prime \bar{i}}-A_{\imath \jmath}=R_{11 j}^{\imath}+\frac{1}{n-2} R_{\imath \jmath}, \quad i \neq j, \\
& K_{1 i}^{\prime \prime \bar{i}}=R_{11 i}^{2}+\frac{1}{n-2} R_{11}-\frac{R}{2(n-1)(n-2)}+\frac{1}{n-2} R_{i i}-\frac{R}{2(n-1)(n-2)} \\
& =R_{11 i}^{2}+\frac{1}{n-2} R_{i i}+\frac{1}{n-2} R_{11}-\frac{R}{(n-1)(n-2)} \text {, } \\
& K_{j k}^{\prime \prime i}=K_{j k}^{\prime \bar{i}}=R_{1 j k}^{2}-\frac{1}{n-2}\left(R_{1 k} \delta_{j}^{i}-R_{1 j} \delta_{k}^{i}\right) \\
& K_{\bar{j} \gamma}^{\prime \prime}=K_{j \gamma}^{\prime \bar{i}}=0 \text {, } \\
& \dot{Q}^{\prime \prime i}-\Omega^{\prime}{ }_{j}=\sum_{k=2}^{n} A_{i k} \theta^{k} \wedge \theta^{j}-\frac{1}{2} \theta^{i} \wedge \theta^{j}+\sum_{k=2}^{n} \theta^{i} \wedge A_{j k} \theta^{k}-\frac{1}{2} \theta^{i} \wedge \theta^{j} \\
& \longleftrightarrow K_{j k 1}^{\prime \prime i}=K_{j k 1}^{\prime i}=R_{j k 1}^{\imath} \text {, } \\
& K^{\prime \prime i k m}=K^{\prime i k m}+A_{i k} \delta_{m}^{j}-A_{\imath m} \delta_{k}^{j}+A_{\jmath m} \delta_{k}^{i}-A_{j k} \delta_{m}^{i}, \\
& =R_{j k m}^{i}-\frac{1}{n-2}\left(R_{i k} \delta_{m}^{\jmath}-R_{\imath m} \delta_{k}^{\jmath}+R_{\jmath m} \delta_{k}^{i}-R_{j k} \delta_{m}^{i}\right) \\
& +\frac{R}{(n-1)(n-2)}\left(\delta_{k}^{i} \delta_{\jmath m}-\delta_{m}^{i} \delta_{j k}\right) \\
& K^{\prime \prime \prime}{ }_{j i j}=K^{\prime i}{ }_{j i j}-1=0 \text {, } \\
& K^{\prime \prime}{ }_{j \beta \gamma}^{i}=K^{\prime i}{ }_{j \beta}=0 \text { otherwise, } \\
& \Omega_{* 0}^{\prime \prime 0}-\Omega_{0}^{\prime 0}=\sum_{\imath, j=2}^{n} \theta^{2} \wedge A_{\imath j} \theta^{\jmath} \\
& \longleftrightarrow K^{\prime \prime 01 j}=K_{01 j}^{\prime 0}=-\frac{1}{2(n-2)} R_{1 j}, \\
& K^{\prime \prime 0}{ }_{0 i j}=K_{0 \imath j}^{\prime 0}+A_{\imath j}-A_{j i}=0 \text {, } \\
& K^{\prime \prime 0}{ }_{0 \beta \gamma}=K_{0 \beta \gamma}^{\prime 0}=0 \text { otherwise, } \\
& \Omega_{1}^{\prime \prime}-\Omega_{1}^{\prime 0}=-\frac{1}{2} \sum_{i=2}^{n} \theta^{i} \wedge \theta^{i}
\end{aligned}
$$


LIE CONTACT STRUCTURES AND CONFORMAL STRUCTURES

61

$$
\begin{aligned}
& \longleftrightarrow K^{\prime \prime 0}{ }_{1 i i}=K^{\prime 0}{ }_{1 i i}-\frac{1}{2}=0, \\
& K^{\prime \prime 1 \beta \gamma}=K^{\prime 0}{ }_{1 \beta \gamma}=0 \text { otherwise, } \\
& \Omega_{0}^{\prime \prime 1}-\Omega_{0}^{\prime 1}=\sum_{i, j=2}^{n} \theta^{i} \wedge A_{\imath j} \theta^{\jmath} \\
& \longleftrightarrow K^{\prime \prime 1}{ }_{0 i \jmath}=K^{\prime 1}{ }_{0 i j}+A_{\imath \jmath}=0 \text {, } \\
& K^{\prime \prime 1}{ }_{01 j}^{1}=K_{01 j}^{\prime 1}=\frac{1}{n-2} \partial_{1} R_{1 j}-\frac{1}{n-2} \partial_{j} R_{11}+\frac{1}{2(n-1)(n-2)} \partial_{j} R, \\
& K^{\prime \prime 1}{ }_{01 j}^{1}=K_{01 j}^{\prime 1}=-\partial_{j} A_{01}^{1}+\frac{1}{n-2} R_{1 j} \text {, } \\
& K^{\prime \prime 1}{ }_{0 \imath \jmath}=K^{\prime 1}{ }_{0 \imath \jmath}=\frac{1}{n-2}\left(\partial_{i} R_{1 j}-\partial_{\jmath} R_{1 \imath}\right) \text {, } \\
& K^{\prime \prime}{ }_{0 i \bar{j}}=K^{\prime 1}{ }_{0 i \bar{j}}=0 \text {, } \\
& \Omega^{\prime \prime 1}-\Omega^{\prime 1}{ }_{1}=-\frac{1}{2} \theta^{i} \wedge \theta^{i}=0 \\
& \longleftrightarrow K^{\prime \prime 1}{ }_{11}^{1}=\frac{1}{2(n-2)} R_{1 \jmath}, \quad K^{\prime \prime 1}{ }_{1 \beta \gamma}=0 \text { otherwise , } \\
& \Omega_{\imath}^{\prime \prime}-\Omega_{i}^{\prime}=d\left(\sum_{j=2}^{n} A_{\imath j} \theta^{j}\right)+A_{i \bar{i}} \theta^{\bar{i}} \wedge\left(\chi_{0}^{\prime 1}+\sum_{j=2}^{n} A_{0 j}^{1} \theta^{\jmath}+A_{01}^{1} \theta^{1}\right) \\
& =\sum_{k=1}^{n} \sum_{j=2}^{n}\left(\partial_{k} A_{\imath \jmath}\right) \theta^{k} \wedge \theta^{\jmath}+\sum_{\jmath, k=2}^{n}\left(\partial_{\bar{k}} A_{\imath \jmath}\right) \theta^{\bar{k}} \wedge \theta^{\jmath}-\sum_{\jmath=2}^{n} A_{\imath j} \theta^{\bar{j}} \wedge \theta^{1} \\
& -\sum_{\jmath=2}^{n} \frac{1}{2(n-2)} R_{1 j} \theta^{\bar{i}} \wedge \theta^{\jmath}-\frac{1}{2} A_{01}^{1} \theta^{\bar{i}} \wedge \theta^{1} \\
& \longleftrightarrow K_{i 1 j}^{\prime \prime}=\partial_{1} A_{\imath \jmath}, \\
& K_{\imath j k}^{\prime \prime}=-\partial_{k} A_{\imath j}+\partial_{j} A_{i k}, \\
& K_{i 1 j}^{\prime \prime}=A_{\imath j}+\frac{1}{2} A_{01}^{1} \delta_{j}^{i} \\
& K_{\imath j \bar{k}}^{\prime \prime}=-\partial_{\bar{k}} A_{\imath j}+\frac{1}{2(n-2)} R_{1 j} \delta_{k}^{2}, \\
& \Omega_{i}^{\prime \prime}-\Omega_{i}^{\prime}=d\left(-\frac{1}{2} \theta^{\bar{i}}\right)+\sum_{j=2}^{n} A_{\imath j} \theta^{\jmath} \wedge\left(\chi_{1}^{\prime 0}+A_{11}^{0} \theta^{1}\right) \\
& =-\frac{1}{2} d \theta^{\bar{\imath}}+\sum_{\jmath=2}^{n} A_{2 \jmath} A_{11}^{0} \theta^{\jmath} \wedge \theta^{1}
\end{aligned}
$$




$$
\begin{aligned}
& \longleftrightarrow K_{\bar{i} 1 j}^{\prime \prime}=-\frac{1}{2} R_{11 j}^{\imath}+\frac{1}{2} A_{\imath \jmath} \\
& K_{i 1 j}^{\prime \prime}=0 \text {, } \\
& K_{i j k}^{\prime \prime}=-\frac{1}{2} R_{1 j k}^{\imath}, \\
& K_{i j \gamma}^{\prime \prime}=K_{i j \gamma}^{\prime}=0 \text {, } \\
& \Omega_{1}^{\prime \prime}-\Omega_{1}^{\prime}=-\sum_{i, j}^{n} A_{\imath j} \theta^{\jmath} \wedge\left(-\frac{1}{2} \theta^{\bar{i}}\right) \\
& \longleftrightarrow K_{11 \jmath}^{\prime \prime}=K_{11 j}^{\prime \prime}=K_{1 \imath \jmath}^{\prime \prime}=K_{1 i j}^{\prime \prime}=0 \text {, } \\
& K_{1 \bar{\imath} \jmath}^{\prime \prime}=-\frac{1}{2} A_{\imath \jmath} \text {. }
\end{aligned}
$$

COROLLARY. Let $C_{j k m}^{\imath}$ and $C_{\imath \jmath k}=\Pi_{\imath \jmath, k}-\Pi_{i k, \jmath}$ be the coefficients of Weyl's conformal curvature tensor at $p=\pi_{1}^{\circ} \pi\left(u_{0}\right) \in M$. Then we have

$$
\begin{array}{ll}
K_{1 j}^{\prime \prime}=C_{11 \jmath}^{\imath}, & K_{j k}^{\prime i}=C_{1 j k}^{\bar{i}}, \\
K_{01 j}^{\prime \prime 1}=C_{11 \jmath}, & K_{0 \imath j}^{\prime 11}=C_{1 \imath \jmath}, \\
K^{\prime \prime i}{ }_{j k m}=C_{j k m}^{\imath}, &
\end{array}
$$

where $2 \leqq i, j, k, m \leqq n$

Proof. Since

$C_{j k m}^{\imath}=R_{j k m}^{\imath}+\frac{1}{n-2}\left(R_{j k} \delta_{m}^{\imath}-R_{\jmath m} \delta_{k}^{2}+g_{j k} R_{m}^{\imath}-g_{\jmath m} R_{k}^{i}\right)-\frac{R}{(n-1)(n-2)}\left(g_{j k} \delta_{m}^{\imath}-g_{\jmath m} \delta_{k}^{i}\right)$, noting $g_{\imath j}(p)=\delta_{i \jmath}$, the last formula follows immediately. Then for $i, j \geqq 2$, we have

$$
\begin{aligned}
C_{11 \jmath}^{\imath} & =R_{11 j}^{\imath}+\frac{1}{n-2}\left(R_{11} \delta_{\jmath}^{i}+R_{\jmath}^{i}\right)-\frac{R}{(n-1)(n-2)} \delta_{\jmath}^{i} \\
& =K_{1 j}^{\prime \prime \bar{i}} .
\end{aligned}
$$

Similarly, we have

Moreover, from

$$
\begin{aligned}
C_{1 j k}^{\imath} & =R_{1 j k}^{\imath}+\frac{1}{n-2}\left(R_{1 j} \delta_{k}^{i}-R_{1 k} \delta_{j}^{i}\right) \\
& =K_{j k}^{\prime \prime \bar{i}} .
\end{aligned}
$$

$$
\Pi_{j k}=-\frac{R_{j k}}{n-2}+\frac{R g_{j k}}{2(n-1)(n-2)},
$$


and $\partial_{k} R_{\imath \jmath}=R_{\imath \jmath, k}, \partial_{k} R=R_{, k}$ at $p$, we get

$$
\begin{aligned}
C_{11 \jmath} & =\Pi_{11, j}-\Pi_{1 \jmath, 1} \\
& =-\frac{1}{n-2} \partial_{j} R_{11}+\frac{1}{2(n-1)(n-2)} \partial_{j} R+\frac{1}{n-2} \partial_{1} R_{1 \jmath} \\
& =K^{\prime \prime 1}{ }_{01 \jmath}^{1},
\end{aligned}
$$

and similarly,

$$
\begin{aligned}
C_{1 \imath \jmath} & =-\frac{1}{n-2} \partial_{j} R_{1 i}+\frac{1}{n-2} \partial_{i} R_{1 \jmath} \\
& =K^{\prime \prime 1}{ }_{0 \imath \jmath} .
\end{aligned}
$$

q.e.d.

\section{$\S 4$. The curvatures and the main result.}

As in $[\mathrm{M} 1, \S 5]$, we can construct Tanaka connection $(P, \omega)$ using Proposition 3.2. For the explicite description of $\omega$, we need some more calculations, but the essential information of the curvature $K$ of $\omega$ is given by its corollaly. In fact, it is shown in [SY] that the harmonic part $H^{p, 2}(K)$ of the curvature $K$ of $\omega$ determines the structure essentially. Moreover, in the case of Lie contact structures, $H^{p, 2}(K)$ vanishes except for $p=0$ if $n \geqq 4$, and $p=0,1$ if $n=3$ [SY]. Therefore it is sufficient to compute $K_{-1}$ for $n \geqq 4$ and $K_{-1}$ and $K_{0}$ for $n=3$. It is easy to see that $K_{-1}^{\prime \prime}=K_{-1}$ [M1]. Immediately, we obtain from Proposition 3.2 and its corollary:

PROPOSITION 4.1. Let $C_{j k m}^{2}$ be the coefficients of Weyl's conformal curvature at $p=\pi_{1} \circ \pi\left(u_{0}\right) \in M, u_{0} \in P_{g}$. Then the curvature $K_{-1}$ of Tanana connection $\omega$ on $\pi: P \rightarrow T_{1} M$ is given by

$$
K_{1 j}^{i}\left(u_{0}\right)=C_{11 j}^{\imath}(p), \quad K_{j k}^{i}\left(u_{0}\right)=C_{1 j k}^{\imath}(p) .
$$

and all other coefficients vanish.

In particular, when $n=3, K_{-1}$ vanishes identically, which is already proved in [SY] from the view point of integrability of $C R$-structures and twistor geometry. Thus in this case, we should compute $K_{0}$. As is shown in [M1], we can see that $K_{01 \jmath}^{1}=K_{01 \jmath}^{\prime 1}$ and $K_{0 \imath \jmath}^{1}=K_{0 \imath \jmath}^{\prime 1}$. Now, we prove:

Proposition 4.2. When $n=3$, by using the coefficients $C_{\imath j k}$ of Weyl's conformal curvature tensors, the curvature $K_{0}$ of Tanaka conection is given by

$$
K_{01 j}^{1}\left(u_{0}\right)=C_{11 j}(p), \quad K_{0 \imath j}^{1}\left(u_{0}\right)=C_{1 \imath j}(p) .
$$

and all other coefficients vanish. 
Proof. We may prove the last statement. For the present, we do not assume $n=3$. Using $(10)^{\prime}$ of $[\mathrm{M} 1, \S 5]$, we have

$$
\begin{aligned}
& -(2 n-1) A_{i l} \\
= & K_{0 i 1}^{\prime \prime 0}+K^{\prime \prime}{ }_{0 \bar{i} 1}-\sum_{k=2}^{n}\left(K_{k k 1}^{\prime \prime i}+K_{i k \bar{k}}^{\prime \prime}\right) \\
= & \frac{1}{2(n-2)} R_{1 i}+\partial_{\bar{i}} A_{01}^{1}-\frac{1}{n-2} R_{1 i}+R_{1 i}-\sum_{k}\left(-\partial_{\bar{k}} A_{i k}+\frac{1}{2(n-2)} R_{1 k} \delta_{k}^{i}\right) \\
= & \frac{n-3}{n-2} R_{1 i}+\partial_{\bar{i}} A_{01}^{1}+\sum_{k=2}^{n} \partial_{\bar{k}} A_{i k} .
\end{aligned}
$$

Now we prepare:

LEMMA 4.3 We have at $u_{0} \in P_{g}$,

$$
\begin{aligned}
& \partial_{\bar{r}} R_{j k m}^{\imath}=-R_{j k m}^{1} \delta_{r i}-R_{1 k m}^{\imath} \delta_{r j}+R_{\jmath r m}^{\imath} \delta_{k 1}-R_{j 1 m}^{\imath} \delta_{r k}+R_{j k r}^{\imath} \delta_{m 1}-R_{j k 1}^{\imath} \delta_{r m}, \\
& \partial_{\bar{r}} R_{11}=2 R_{1 r}, \\
& \partial_{\bar{r}} A_{01}^{1}=\frac{2}{n-2} R_{1 r}, \\
& \partial_{\bar{r}} A_{\imath \jmath}=\frac{1}{n-2}\left(R_{1 j} \delta_{r i}+R_{1 i} \delta_{r \jmath}\right),
\end{aligned}
$$

where $2 \leqq r \leqq n, 1 \leqq i, j, k, m \leqq n$.

Proof. Since we have $R_{j k m}^{2}=\Psi^{\prime i}{ }_{j k m}, 2 \leqq i, j \leqq n, 1 \leqq k, m \leqq n$, from

$$
\begin{aligned}
\partial_{\bar{r}} \Psi_{j}^{\prime i}= & \partial_{\bar{r}} d\left(g_{u v} z_{i}^{v}\left(d z_{\jmath}^{u}+\left\{\begin{array}{c}
u \\
s t
\end{array}\right\} z_{j}^{s} d x^{t}\right)\right) \\
\equiv & \partial_{\bar{r}}\left(\partial_{h}\left(g_{u v} z_{i}^{v}\left\{\begin{array}{c}
u \\
s t
\end{array}\right\} z_{\jmath}^{s}\right) z_{k}^{h} z_{m}^{t}\right) \zeta^{k} \wedge \zeta^{m} \quad\left(\bmod \zeta^{\bar{k}} \wedge \zeta^{r}\right) \\
= & \left\{\left(\partial_{h}\left\{\begin{array}{c}
v \\
s t
\end{array}\right\}\right) \partial_{\bar{r}}\left(z_{i}^{v} z_{\jmath}^{s}\right) \delta_{k}^{h} \delta_{m}^{t}+\partial_{h}\left\{\begin{array}{c}
i \\
j t
\end{array}\right\} \partial_{\bar{r}}\left(z_{k}^{h} z_{m}^{t}\right)\right\} \zeta^{k} \wedge \zeta^{m} \\
= & {\left[\partial_{k}\left\{\begin{array}{c}
v \\
s m
\end{array}\right\}\left\{\left(\delta_{r}^{v} \delta_{i 1}-\delta_{r i} \delta_{1}^{v}\right) \delta_{j}^{s}+\delta_{i}^{v}\left(\delta_{r}^{s} \delta_{j 1}-\delta_{r j} \delta_{1}^{s}\right)\right\}\right.} \\
& \left.+\partial_{h}\left\{\begin{array}{c}
i \\
j t
\end{array}\right\}\left(\delta_{r}^{h} \delta_{k 1}-\delta_{r k} \delta_{1}^{h}\right) \delta_{m}^{t}+\delta_{k}^{h}\left(\delta_{r}^{t} \delta_{m 1}-\delta_{r m} \delta_{1}^{t}\right)\right] \zeta^{k} \wedge \zeta^{m} \\
= & {\left[-\partial_{k}\left\{\begin{array}{c}
1 \\
j m
\end{array}\right\} \delta_{r i}-\partial_{k}\left\{\begin{array}{c}
i \\
1 m
\end{array}\right\} \delta_{r j}+\partial_{r}\left\{\begin{array}{c}
i \\
j m
\end{array}\right\} \delta_{k 1}\right.}
\end{aligned}
$$




$$
\left.-\partial_{1}\left\{\begin{array}{c}
i \\
j m
\end{array}\right\} \delta_{r k}+\partial_{k}\left\{\begin{array}{c}
i \\
j r
\end{array}\right\} \delta_{m 1}-\partial_{k}\left\{\begin{array}{c}
i \\
j 1
\end{array}\right\} \delta_{r m}\right] \zeta^{k} \wedge \zeta^{m}
$$

we get

$$
\partial_{\bar{r}} R_{j k m}^{\imath}=-R_{j k m}^{1} \delta_{r i}-R_{1 k m}^{\imath} \delta_{r j}+R_{\jmath r m}^{\imath} \delta_{k 1}-R_{j 1 m}^{\imath} \delta_{r k}+R_{j k r}^{\imath} \delta_{m 1}-R_{j k 1}^{\imath} \delta_{r m} .
$$

Therefore, we obtain

for $j, m \geqq 2$, and

$$
\begin{aligned}
\partial_{\bar{r}} \tilde{R}_{\jmath m} & =\sum_{\imath=2}^{n} \partial_{\bar{r}} R_{j \imath m}^{\imath} \\
& =-R_{\jmath r m}^{1}-R_{1 m} \delta_{r j}-R_{j 1 m}^{r}-R_{\jmath 1} \delta_{r m}
\end{aligned}
$$

$$
\begin{aligned}
\partial_{\bar{r}} \tilde{R} & =\sum_{\jmath=2}^{n} \partial_{\bar{r}} \widetilde{R}_{\jmath \jmath} \\
& =-4 R_{1 r} .
\end{aligned}
$$

Now, using (2.13), we have

$$
\partial_{\bar{r}} R_{11}=-\sum_{m=2}^{n} \partial_{\bar{r}} T_{1 m}^{\prime \bar{m}}=-\left(-R_{1 r}-R_{1 r}\right)=2 R_{1 r} .
$$

Since $R$ is a function on $M, \partial_{\bar{r}} R=0$ is trivial, but also follows from

and so we obtain

$$
\partial_{\bar{r}} R=\partial_{\bar{r}}\left(\tilde{R}+2 R_{11}\right)=0,
$$

$$
\begin{aligned}
\partial_{\bar{r}} A_{01}^{1} & =\frac{1}{n-2} \partial_{\bar{r}} R_{11}-\frac{1}{2(n-1)(n-2)} \partial_{\bar{r}} R \\
& =\frac{2}{n-2} R_{1 r} .
\end{aligned}
$$

Moreover, using (2.13) and (3.2), we get

$$
\begin{aligned}
\partial_{\bar{r}} A_{\imath \jmath} & =-\frac{1}{n-2} \partial_{\bar{r}}\left(R_{1 j 1}^{\imath}+\widetilde{R}_{\imath \jmath}\right)+\frac{1}{2(n-1)(n-2)} \partial_{\bar{r}} R \delta_{\jmath}^{\imath} \\
& =-\frac{1}{n-2}\left(R_{r j 1}^{\imath}+R_{1 \jmath r}^{\imath}-R_{\imath r j}^{1}-R_{1 j} \delta_{r i}-R_{i 1 j}^{r}-R_{i 1} \delta_{r \jmath}\right) \\
& =\frac{1}{n-2}\left(R_{1 j} \delta_{r i}+R_{1 i} \delta_{r \jmath}\right) .
\end{aligned}
$$

Finally, we obtain from (4.1),

$$
A_{i 1}=-\frac{1}{n-2} R_{1 \imath} \text {. }
$$

Next, the left hand sides of $(11)^{\prime}$ and $(13)^{\prime}$ of $[\mathrm{M} 1, \S 5]$ are zero and we get 


$$
A_{\bar{i}_{1}}=A_{1 j}=0 \text {. }
$$

From $(12)^{\prime}$, we have

$$
\begin{aligned}
\left(K_{01 j}^{\prime \prime 0}+K_{11 \jmath}^{\prime 1}\right)-\sum_{i=2}^{n}\left(K_{i i j}^{\prime \prime}-K_{i i \jmath}^{\prime \prime}\right)= & -\frac{1}{2(n-2)} R_{1 j}+\frac{1}{2(n-2)} R_{1 \jmath} \\
& +\frac{1}{2} R_{1 j}+\frac{n+1}{2(n-2)} R_{1 \jmath} \\
= & \frac{2 n-1}{2(n-2)} R_{1 \jmath},
\end{aligned}
$$

so that

$$
A_{1 \jmath}=-\frac{1}{2(n-2)} R_{1 \jmath} .
$$

Using (3.1), we obtain

$$
\begin{aligned}
& K_{j k 1}^{i}=K_{j k 1}^{\prime \prime i}-A_{i 1} \delta_{k}^{j}+A_{j 1} \delta_{k}^{i} \\
& =R_{j k 1}^{\imath}+\frac{1}{n-2}\left(R_{1 i} \delta_{k}^{j}-R_{1 j} \delta_{k}^{i}\right)=C_{j k 1}^{\imath}, \\
& K_{j k m}^{\imath}=K_{j k m}^{\prime \prime i}=C_{j k m}^{\imath} \text {, } \\
& K_{j \beta \gamma}^{i}=K_{j \beta \gamma}^{\prime i}=0 \quad \text { otherwise, } \\
& K_{0 i 1}^{0}=K_{0 i 1}^{\prime \prime 0}+A_{i 1}-A_{1 \imath} \\
& =\left\{\frac{1}{2(n-2)}-\frac{1}{n-2}+\frac{1}{2(n-2)}\right\} R_{12}=0, \\
& K_{0 \beta \gamma}^{0}=K^{\prime \prime 1}{ }_{0 \beta \gamma}^{0}=0 \quad \text { otherwise, } \\
& K_{1 \beta \gamma}^{0}=K_{1 \beta \gamma}^{\prime \prime 0}=0 \text {, } \\
& K_{01 j}^{1}=K^{\prime 11}{ }_{01 j}=C_{11 j} \\
& K_{0 \imath j}^{1}=K^{\prime 1}{ }_{0 \imath \jmath}^{1}=C_{1 \imath \jmath} \text {, } \\
& K_{01 j}^{1}=K_{01 j}^{\prime \prime 1}-A_{j 1}=-\partial_{j} A_{01}^{1}+\frac{1}{n-2} R_{1 j}+\frac{1}{n-2} R_{1 \jmath}=0 \text {, } \\
& K_{0 \beta \gamma}^{1}=K_{0 \beta \gamma}^{11}=0 \quad \text { otherwise, } \\
& K_{1 i 1}^{1}=K^{\prime \prime 1}{ }_{1 i 1}-A_{12}=\left\{-\frac{1}{2(n-2)}+\frac{1}{2(n-2)}\right\} R_{12}=0 \text {, } \\
& K_{1 \beta \gamma}^{1}=K_{1 \beta \gamma}^{\prime 1}=0 \quad \text { otherwise. }
\end{aligned}
$$

Since when $n=3$ we have

$$
C_{j k m}^{\imath}=0,
$$


the proposition is proved.

q.e.d.

From the property C3) of Cartan connections, or, since $K$ is a tensorial 2 form of type $(A d, g)$ on $P$, the harmonic part of $K$ is determined all over $P$ by Proposition 4.1 and 4.2. Moreover, noting that the index 1 in Proposition 4.1 and 4.2 denotes the direction of the base point $\pi\left(u_{0}\right) \in T_{1} M$, which is arbitrarily chosen, we obtain the main results, Corollary 1 and 2 .

Since we have used here the fact described in the beginning of this section, we will give a direct proof of these results in the next section, to be selfcontained.

\section{$\S 5$. Tanaka connection and the Lie curvature in a local coordinate.}

In order to give a complete description of $\omega$, determine $A_{11}$ by $(14)^{\prime}$ of [M1]. We prepare first

LEMMA 5.1. We have at $u_{0} \in P_{g}$,

$$
\begin{aligned}
& \partial_{j} A_{i 1}=-\frac{1}{n-2}\left(R_{\imath j}-R_{11} \delta_{i \jmath}\right), \\
& \partial_{j} A_{12}=-\frac{1}{2(n-2)}\left(R_{\imath j}-R_{11} \delta_{\imath \jmath}\right) .
\end{aligned}
$$

Proof. This follows easily from (2.13) and

$$
\begin{aligned}
\partial_{j} R_{1 \imath} & =-\partial_{j} \sum_{m=2}^{n} T_{\imath m}^{\prime \bar{m}}=\widetilde{R}_{\imath \jmath}-R_{11} \delta_{i j}+R_{1 i 1}^{\jmath} \\
& =R_{\imath j}-R_{11} \delta_{i \jmath} .
\end{aligned}
$$

Now, we obtain

$$
\begin{aligned}
\sum_{i=2}^{n}\left(\partial_{\bar{i}} A_{i 1}+\partial_{\bar{i}} A_{1 \imath}\right) & =-\left\{\frac{1}{n-2}+\frac{1}{2(n-2)}\right\} \sum_{i=2}^{n} \partial_{\bar{i}} R_{1 \imath} \\
& =-\frac{3}{2(n-2)}\left\{\sum_{i=2}^{n} R_{i i}-(n-1) R_{11}\right\} \\
& =-\frac{3}{2(n-2)}\left(R-n R_{11}\right),
\end{aligned}
$$

and

$$
\begin{aligned}
\sum_{i=2}^{n}\left(-K_{i i 1}^{\prime \prime}+K_{i \overline{1} 1}^{\prime \prime}-K_{1 i \bar{i}}^{\prime \prime}\right) & =\frac{1}{2} R_{11}+\frac{1}{2} \sum_{i=2}^{n} A_{i i}-\sum_{i=2}^{n} A_{i i}-\frac{1}{2}(n-1) A_{01}^{1}-\frac{1}{2} \sum_{i=2}^{n} A_{i i} \\
& =\frac{1}{2} R_{11}+\frac{1}{n-2} \sum_{i=2}^{n} R_{i i}-\frac{R}{2(n-2)}-\frac{n-1}{2(n-2)} R_{11}+\frac{R}{4(n-2)}
\end{aligned}
$$




$$
=-\frac{3}{2(n-2)} R_{11}+\frac{3 R}{4(n-2)} \text {. }
$$

Thus, we obtain

$$
A_{11}=-\frac{1}{2(n-2)} R_{11}+\frac{R}{4(n-1)(n-2)} .
$$

As for the curvature $\Omega=1 / 2 K \theta \wedge \theta$ of $\omega$, the $g_{p}$-components $K_{p}$ is given in Proposition 3.2 for $p<0$, and in the proof of Proposition 4.2 for $p=0$. For $p>0$, we have from

$$
\begin{aligned}
& \Omega_{i}-\Omega_{\imath}^{\prime \prime}=d\left(A_{i 1} \theta^{1}\right)+\theta^{\bar{\imath}} \wedge \sum_{j=2}^{n} A_{1 j} \theta^{\jmath}+\theta^{\bar{i}} \wedge A_{11} \theta^{1}, \\
& \longleftrightarrow K_{i 1 \jmath}=K_{i 1 j}^{\prime \prime}-\partial_{\jmath} A_{i 1} \\
& =\partial_{1} A_{\imath j}-\partial_{\jmath} A_{i 1}=\Pi_{\imath \jmath, 1}-\Pi_{i 1, \jmath}=C_{\imath j 1}, \\
& K_{i 1 j}=K_{i 1 j}^{\prime \prime} \partial_{j} A_{i 1}-A_{11} \delta_{j}^{2} \\
& =-\frac{1}{n-2} R_{\imath j}+\frac{R}{2(n-1)(n-2)} \delta_{j}^{\imath}+\frac{1}{2(n-2)}\left\{R_{11}-\frac{R}{2(n-1)}\right\} \delta_{j}^{\imath} \\
& +\frac{1}{n-2}\left(R_{\imath j}-R_{11} \delta_{\jmath}^{i}\right)-\left\{-\frac{1}{2(n-2)} R_{11}+\frac{R}{4(n-1)(n-2)}\right\} \delta_{\jmath}^{i} \\
& =0 \text {, } \\
& K_{\imath j k}=K_{\imath j k}^{\prime \prime}=-C_{\imath j k} \text {, } \\
& K_{\imath j k}=K^{\prime \prime}{ }_{j k}+A_{i 1} \delta_{k}^{\jmath}+A_{1 k} \delta_{j}^{2} \\
& =\frac{1}{n-2}\left(R_{1 i} \delta_{k}^{\jmath}+R_{1 k} \delta_{\jmath}^{i}\right)-\frac{1}{2(n-2)} R_{1 k} \delta_{\jmath}^{2}-\frac{1}{n-2} R_{1 i} \delta_{k}^{\jmath}-\frac{1}{2(n-2)} R_{1 k} \delta_{\jmath}^{\imath} \\
& =0 \text {, } \\
& K_{i j \bar{k}}=K^{\prime \prime}{ }^{j \bar{k}}=0 .
\end{aligned}
$$

Similarly, we obtain

$$
\begin{aligned}
\Omega_{\bar{i}}-\Omega^{\prime \prime}{ }_{i} & =-\theta^{\imath} \wedge\left(\sum_{j=2}^{n} A_{1 j} \theta^{\jmath}+A_{11} \theta^{1}\right) \\
\longleftrightarrow K_{i 1 j} & =K^{\prime \prime}{ }_{i j j}+A_{11} \delta_{\jmath}^{2} \\
= & -\frac{1}{2} R_{11 j}^{\imath}-\frac{1}{2(n-2)} R_{\imath j}+\frac{R}{4(n-1)(n-2)} \delta_{\jmath}^{i} \\
& -\frac{1}{2(n-2)} R_{11}+\frac{R}{4(n-1)(n-2)} \delta_{\jmath}^{2}
\end{aligned}
$$




$$
\begin{aligned}
& =-\frac{1}{2} C_{11 \jmath}^{2}, \\
& K_{\bar{i} 1 j}=K_{\bar{i}_{1 j}}^{\prime \prime}=0 \text {, } \\
& K_{i j k}=K_{i j k}^{\prime \prime}-A_{1 k} \delta_{j}^{2}+A_{1 j} \delta_{k}^{2} \\
& =-\frac{1}{2} R_{1 j k}^{\imath}+\frac{1}{2(n-2)} R_{1 k} \delta_{j}^{2}-\frac{1}{2(n-2)} R_{1 j} \delta_{k}^{\imath} \\
& =-\frac{1}{2} C_{1 j k}^{2}, \\
& K_{\bar{i}{ }_{k}}=K_{\bar{i}_{j k}}^{\prime \prime}=0 \text {, } \\
& \Omega_{1}-\Omega_{1}^{\prime \prime}=d\left(\sum_{\jmath=2}^{n} A_{1 \jmath} \theta^{\jmath}+A_{11} \theta^{1}\right) \sum_{\imath=2}^{n} A_{\imath 1} \theta^{1} \wedge A_{i \bar{\imath}} \theta^{\bar{\imath}} \\
& \longleftrightarrow K_{11 \jmath}=\partial_{1} A_{1 j}-\partial_{\jmath} A_{11}=\frac{1}{2} C_{1 \jmath 1}, \\
& K_{11 j}=A_{1 j}-\partial_{j} A_{11}-A_{j 1} A_{j j} \\
& =-\frac{1}{2(n-2)} R_{1 j}+\frac{1}{2(n-2)} \partial_{j} R_{11}-\frac{1}{2(n-2)} R_{1 j} \\
& =-\frac{1}{n-2} R_{1 j}+\frac{1}{n-2} R_{1 j}=0 \text {, } \\
& K_{1 j k}=\partial_{\jmath} A_{1 k}-\partial_{k} A_{1 \jmath}=-\frac{1}{2} C_{1 \jmath k} \text {, } \\
& K_{1 j \bar{k}}=K_{1 j \bar{k}}^{\prime \prime}-\partial_{\bar{k}} A_{1 j}-A_{11} \delta_{k}^{j} \\
& =\frac{1}{2}\left\{-\frac{1}{n-2} R_{j k}+\frac{R}{2(n-1)(n-2)} \delta_{k}^{\jmath}\right\}+\frac{1}{2(n-2)}\left(R_{j k}-R_{11} \delta_{j}^{i}\right) \\
& -\left\{-\frac{1}{2(n-2)} R_{11}+\frac{R}{4(n-1)(n-2)}\right\} \delta_{k}^{J} \\
& =0 \text {, } \\
& K_{1 j \bar{k}}=0 \text {, }
\end{aligned}
$$

Now, we summarize our results as a theorem.

THEOREM. Let $\left(x^{2}, z_{j}^{2}, s_{b}^{a}, s_{\imath}, s_{i}, s_{1}\right)$ be the local cordinate around $u_{0} \in P_{g}$ chosen as in $\S 3$. Then at $u=u(z)=\left(x^{2}, z_{\jmath}^{2}, \delta_{b}^{a}, \mathbf{0}, \mathbf{0}, 0\right) \in P_{g}$, Tanaka connection $\omega$ on $\pi: P \rightarrow T_{1} M$ is given by

$$
\theta^{\imath}=g_{j k} z_{i}^{k} d x^{\jmath}, \quad 1 \leqq i \leqq n,
$$




$$
\begin{aligned}
& \theta^{i}=g_{j k} z_{i}^{k}\left(d z_{1}^{j}+\left\{\begin{array}{c}
j \\
s t
\end{array}\right\} z_{1}^{s} d x^{t}\right), \quad 2 \leqq i \leqq n, \\
& \omega_{j}^{2}=g_{u v} z_{i}^{v}\left(d z_{j}^{u}+\left\{\begin{array}{c}
u \\
s t
\end{array}\right\} z_{j}^{s} d x^{t}\right), \quad 2 \leqq i, j \leqq n, \\
& \omega_{0}^{0}=d s_{0}^{0}, \\
& \omega_{1}^{0}=d s_{1}^{0}+A_{11}^{0} \theta^{1}, \\
& \omega_{0}^{1}=d s_{0}^{1}+\sum_{j=2}^{n} A_{0 j}^{1} \theta^{\jmath}+A_{01}^{1} \theta^{1}, \\
& \omega_{1}^{1}=d s_{1}^{1}, \\
& \omega_{i}=d s_{i}+\sum_{j=2}^{n} A_{\imath j} \theta^{j}+A_{\imath 1} \theta^{1}, \\
& \omega_{i}=d s_{i}+A_{i \bar{i}} \theta^{\bar{i}}, \quad 2 \leqq i \leqq n, \\
& \omega_{1}=d s_{1}+\sum_{j=2}^{n} A_{1 j} \theta^{\jmath}+A_{11} \theta^{1},
\end{aligned}
$$

where

$$
\begin{aligned}
& A_{11}^{0}=-\frac{1}{2}, \quad A_{0 \jmath}^{1}=\frac{1}{n-2} R_{1 \jmath}, \quad A_{01}^{1}=\frac{1}{n-2} R_{11}-\frac{R}{2(n-1)(n-2)}, \\
& A_{\imath \jmath}=-\frac{1}{n-2} R_{\imath j}+\frac{R}{2(n-1)(n-2)} \delta_{\jmath}^{\imath}, \quad A_{i i}=-\frac{1}{2}, \\
& A_{i 1}=-\frac{1}{n-2} R_{1 \imath}, \quad A_{1 \imath}=-\frac{1}{2(n-2)} R_{1 \imath}, \\
& A_{11}=-\frac{1}{2(n-2)} R_{11}+\frac{R}{4(n-1)(n-2)},
\end{aligned}
$$

using the Ricci curvature $R_{\imath \jmath}=z_{\imath}=z_{i}^{k} \partial / \partial x_{k}$, and the scalar curvature $R$ of $M$ at $\left(x^{i}\right)$. The curvature $K$ of $\omega$ at $u_{0}$ is given by

$$
\begin{aligned}
& K_{1 \jmath}^{i}=C_{11 \jmath}^{\imath}, \quad K_{j k}^{i}=C_{1 j k}^{\imath}, \\
& K_{01 \imath}^{1}=C_{11 \imath}, \quad K_{0 \imath \jmath}^{1}=C_{1 \imath \jmath}, \quad K_{j k 1}^{i}=C_{j k 1}^{\imath}, \quad K_{j k m}^{i}=C_{j k m}^{\imath}, \\
& K_{i 1 \jmath}=-C_{i 1 \jmath}, \quad K_{\imath j k}=-C_{\imath j k}, \\
& K_{i 1 \jmath}=-\frac{1}{2} C_{11 \jmath}^{\imath}, \quad K_{i j k}=-\frac{1}{2} C_{1 j k}^{\imath}, \\
& K_{11 \jmath}=-\frac{1}{2} C_{11 \jmath}, \quad K_{1 j k}=-\frac{1}{2} C_{1 j k},
\end{aligned}
$$


for $2 \leqq i, j, k, m \leqq n$, and all other components vanish, where $C_{j k m}^{2}$ and $C_{i j k}$ are the coefficients of Weyl's conformal curvature.

Since $R_{a}^{*} \omega=A d\left(a^{-1}\right) \omega, a \in G^{\prime}, \omega$ and $K$ are determined all over $P$ by this theorem.

\section{REFERENCES}

[C C] Cecil, T. and Chern, S.S., Tautness and Lie sphere geometry, Math. Ann., 278 (1987), 381-399.

[M1] MryaokA, R., Lie contact structures and normal Cartan connections, Kodai Math. J. 14 (1991), 13-41.

[M2] MiyaokA, R., A note on Lie contact manifolds, Preprint (1990), Tokyo Institute of Technology.

[O] Ogiue, K., Theory of conformal connections, Kodai Math. Sem. Rep. 19 (1967), 193-224.

[S] SATo, H., Lie's contact geometry and Lie manifolds (in Japanese), Proc. of 35th Top Symp. (1987), Ryukyu Univ., 41-63.

[S Y] Sato, H. And Yamaguchi, K., Lie contact manifolds, "Geometry of manifolds (ed. by K. Shiohama)", Acad. Press (1989), 191-238.

[S S] SASAKI, S., On the differential geomtry of tangent bundles of Riemannian manifolds, Tohoku Math. J., 10 (1958), 338-354.

[T] TANAKA, N., On the equivalence problems associated with graded Lie algebras, Hokkaido Math. J., 8 (1979), 23-84.

Department of Mathematics

TOKYo INSTITUTE OF TECHNOLOGY

Ohokayama, Meguroku, 152

TOKYO/JAPAN 